\title{
Statements on nonlinear dynamics behavior of a pendulum, excited by a crank-shaft-slider mechanism
}

\author{
Rafael Henrique Avanço $\mathbb{D}^{-}$Hélio Aparecido Navarro • \\ Reyolando M. L. R. F. Brasil • José Manoel Balthazar • \\ Átila Madureira Bueno • Angelo Marcelo Tusset
}

Received: 11 May 2015/Accepted: 22 October 2015/Published online: 27 October 2015

(C) Springer Science+Business Media Dordrecht 2015

\begin{abstract}
The nonlinear dynamics behavior analyzed, in this paper, consists in a pendulum vertically excited on the support by a crank-shaft-slider mechanism. The novelty is the obtainment and analysis of a mathematical model for the pendulum dynamics, under an excitation of a crank-slider, which is based on an extension of the mathematical model of the classical parametric pendulums. Through the modeling, it was verified that the nonlinear dynamics of the pendulum, excited by the crank-shaft-slider mechanism approaches to that of harmonic excitation, when one considered the length of the shaft is sufficient larger than the radius of the crank. The nonlinear dynamic analyses focused on observation of different
\end{abstract}

R. H. Avanço $(\square) \cdot$ H. A. Navarro

Department of Mechanical Engineering, University of São Paulo, Av. Trabalhador São-Carlense, n ${ }^{\circ}$ 400, São Carlos, SP 13566-590, Brazil

e-mail: avancorafael@gmail.com

H. A. Navarro

e-mail: han@sc.usp.br

R. M. L. R. F. Brasil

Federal University of ABC, Santa Adélia Street, $n^{\circ}$ 166, Santo André, SP, Brazil

e-mail: reyolando.brasil@ufabc.edu.br

\section{J. M. Balthazar}

Department of Mechanical Engineering at Technological Institute of Aeronautics, Pça. Mal. Eduardo Gomes, ${ }^{\circ}$ 50, São José Dos Campos, SP 12228-900, Brazil

e-mail: jmbaltha@gmail.com kinds of motion for different values of dimensionless parameters of the adopted mathematical model. These parameters, includes the frequency of excitation, the amplitude and the geometry of the crank-shaft-slider mechanism. The adopted method of analyses used tools, such as, Lyapunov exponents, parameter space plots, basins of attractions, bifurcation diagrams, phase portraits, time histories and Poincaré sections. The kinds of motion include results on fixed point, oscillations, rotations, oscillations-rotations and chaotic motions.

Keywords Pendulum - Parametric $\cdot$ Crank-shaftslider $\cdot$ Chaos

\footnotetext{
Á. M. Bueno

UNESP: Sorocaba, Control and Automation Engineering, Av. Três de Março, Sorocaba, SP 18087-180, Brazil e-mail: atila@sorocaba.unesp.br
}
A. M. Tusset
Department of Mathematics, Federal University of Technology - Paraná, Ponta Grossa, PR, Brazil e-mail: brazil.tusset@utfpr.edu.br 


\section{List of symbols}

$a \quad$ Length of the crank rod

$b \quad$ Length of the shaft

$\varepsilon \quad a$ over $b$ length ratio

$m$ Mass of pendulum

$t \quad$ Time

$l \quad$ Length of the pendulum

$\varphi \quad$ Angle between $b$ bar and vertical axis

$\theta \quad$ Angle of the crank rod

$\alpha \quad$ Pendulum rotation angle

$\tau \quad$ Dimensionless time

$\omega \quad$ Ratio between excitation and natural frequency

$\omega_{0} \quad$ Natural frequency of the pendulum

$\lambda$ Maximum Lyapunov exponent

$x_{p} \quad$ Position of pendulum in $\mathrm{x}$ coordinate

$y_{p} \quad$ Position of pendulum in y coordinate

$F \quad$ Dimensionless parameter relating the angles $\theta$ and $\varphi$

\section{Introduction}

The classical parametrically excited pendulum has been vastly studied in the literature and it is verified that the harmonic excitation on the pendulum might provoke different kinds of motion in a simple pendulum. This paper is concerned on a new model of the dynamics of a parametric pendulum, excited vertically on the pivot by a crank-shaft-slider mechanism.

In the past, it was observed that the periodic excitation might lead to chaotic motions in the parametric pendulum in Leven and Koch [1], where the chaotic behavior appears after cascading bifurcations, characterized by an appearance of a strange attractor in the Poincaré section.

Rotating periodic motions were shown in Clifford and Bishop [2] where it was found that we may be able to achieve rotating solutions reducing the force applied in a harmonic stable solution. In Clifford and Bishop [3] the location of oscillatory orbits was found with odd oscillatory periodic solutions through a horseshoe structure formed by invariant manifolds of two saddles.

$\mathrm{Xu}$ et al. [4] brought the idea of rotating periodic motions explored in the sense of generate electricity from the parametrically excited pendulum. The concept of energy extraction from sea waves with the parametric pendulum resulted in a patent application
[5]. A possible utility for the mechanism studied in the present paper is also the energy extraction, although it is enough when you use the classic parametric pendulum for the energy extraction from the sea. The new possibility is a conversion of rotation, i.e., the rotation of the crank converted in the rotation of the pendulum. A crank powered by the wind can produce energy through the rotating of the pendulum. In Fig. 1, the mechanism containing the crank-shaft-slider and the pendulum is sketched.

In Brasil et al. [6], a crank-shaft-slider mechanism without the pendulum is proposed for harvest energy from sea waves. There is a slider which is a floating body free to undergo vertical motions as excited by sea waves where the crank is powered by this slider.

In Kecik and Warminski [7] two kinds of chaotic motions are detected. They are the chaotic swings and the chaotic motion constituted by swings and rotations of the pendulum. The mechanism with a mass linked to ground with a spring and damper shaking a pendulum provided experimental results to confirm the two kinds of chaotic motions.

An analysis involving a comparison between experimental and theoretical robustness of rotating solutions in a parametrically excited pendulum was performed in Lenci and Rega [8]. The experimental results demonstrated that rotations exist in a region

Fig. 1 Schematic diagram of pendulum coupled to the crank-slider mechanism

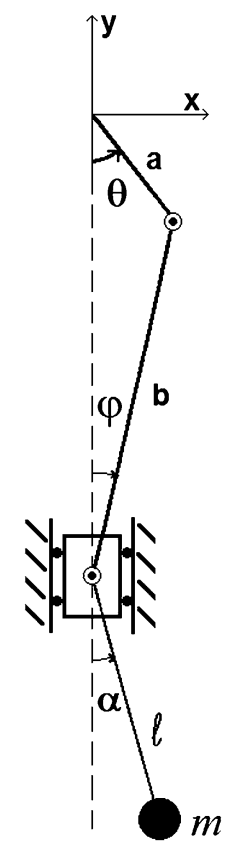



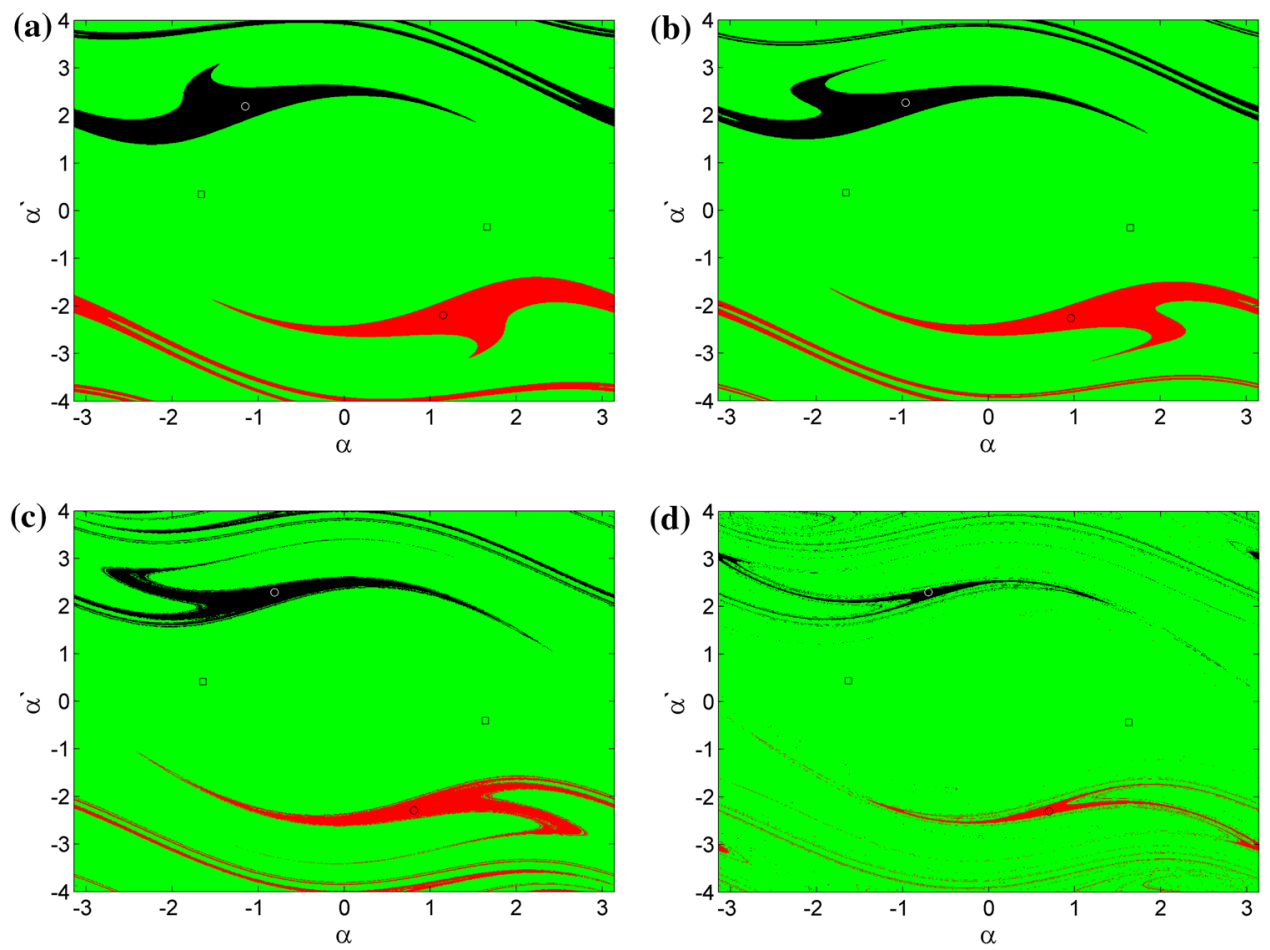

Fig. 2 Basins of attraction for parameters: $p=0.5, \omega=1.8$ and $\gamma=0.1$. a $\varepsilon=0, \mathbf{b} \varepsilon=0.4$, $\mathbf{c} \varepsilon=0.7$, d $\varepsilon=0.9$

Table 1 Position of the attractors $\left(\alpha, \alpha^{\prime}\right)$ for different values of $\varepsilon$

\begin{tabular}{llll}
\hline & Oscillation period-2 & Positive rotating & Negative rotating \\
\hline$\varepsilon=0$ & $(-1.66 ; 0.34)$ and $(1.66 ;-0.34)$ & $(-1.15 ; 2.19)$ & $(1.15 ;-2.19)$ \\
$\varepsilon=0.4$ & $(-1.65 ; 0.37)$ and $(1.65 ;-0.37)$ & $(-0.96 ; 2.26)$ & $(0.96 ;-2.26)$ \\
$\varepsilon=0.7$ & $(-1.64 ; 0.41)$ and $(1.64 ;-0.41)$ & $(-0.81 ; 2.29)$ & $(0.81 ;-2.29)$ \\
$\varepsilon=0.9$ & $(-1.63 ; 0.44)$ and $(1.63 ;-0.44)$ & $(-0.70 ; 2.30)$ & $(0.70 ;-2.30)$ \\
\hline
\end{tabular}

which is smaller than the theoretical one. The reason is that a birth of a highly periodic rotation within the basin of attraction suddenly reduces its safe region.

In Warminski and Kecik [9], it was carried out an analysis of a system including a pendulum, a harmonic oscillating mass in the support, a damper and a nonlinear spring linking to the ground. It has been verified that the motion of the oscillator, forced by an external harmonic force, has been dynamically eliminated by the pendulum oscillations. The influence of the nonlinear spring on the vibration absorption near the main parametric resonance region has been performed analytically.

An analysis of instabilities of an autoparametric system is present in Warminski and Kecik [10]. A nonlinear oscillator and a damped pendulum are investigated in the main resonance. Analytical solutions were obtained based on the Harmonic Balance Method (HBM). The accuracy of the analytical results was confirmed through numerical and experimental results.

Using a perturbation method, the rotation solutions were studied in Lenci et al. [11] considering the undamped unforced Hamiltonian case as a basic solution. The damping and excitation are small perturbations. It was concluded the analytical method is an accurate predictor for large amplitudes.

In Wang and Jing [12] it was implemented the Lyapunov function method for projecting a controller to a pendulum system with chaotic motion to a desired periodic motion like period-1, period- 2 and period- 4 . The major advantage pointed in [11] was the time 

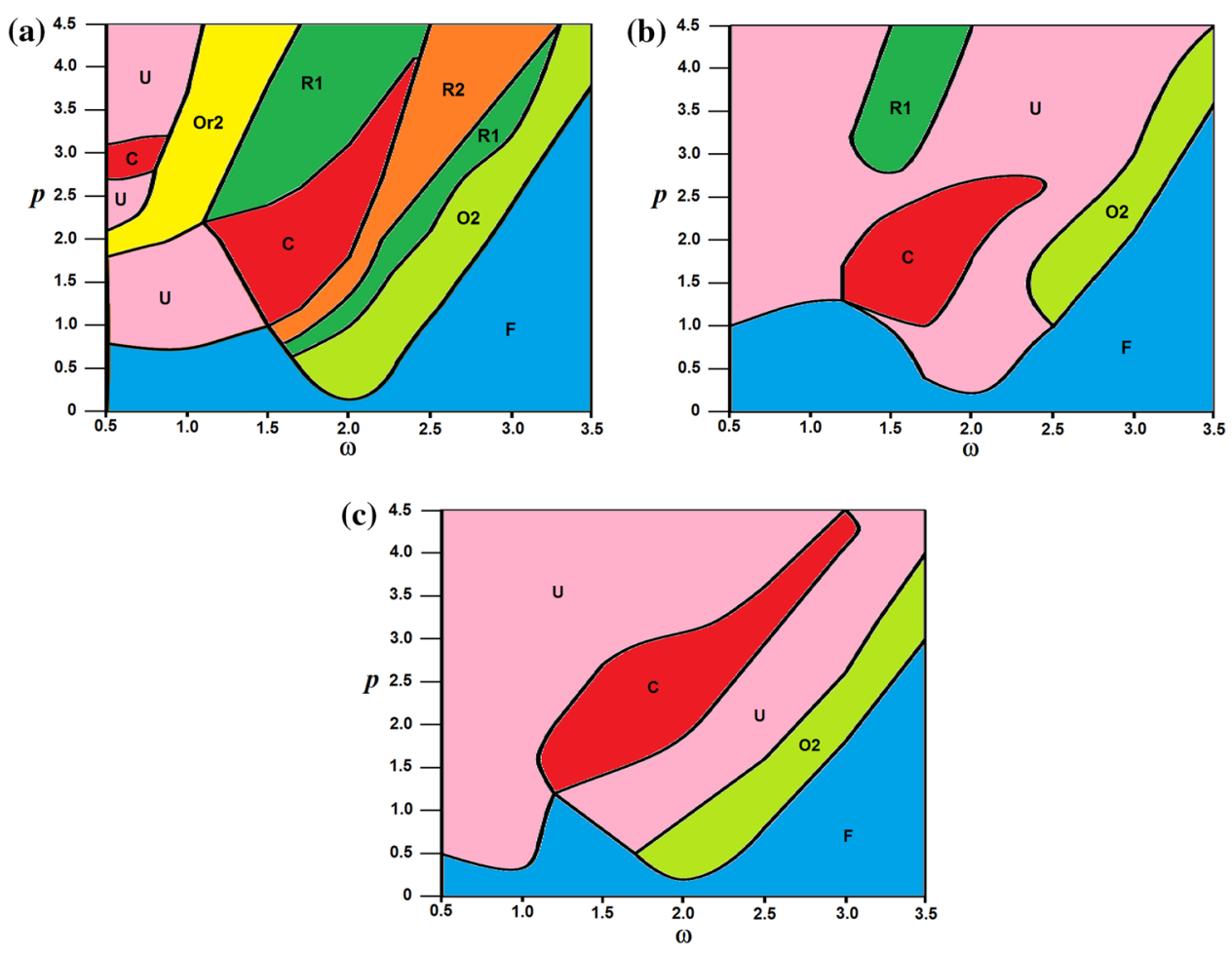

Fig. 3 Parameter space plot $\mathbf{a} \varepsilon=0, \mathbf{b} \varepsilon=0.4$, $\mathbf{c} \varepsilon=0.9$

consumption of control, which is shorter than the OGY and SOGY methods.

It was proposed a control method for establishing a periodic rotation in parametric pendulum based on a delayed feedback control in Yokoi and Hikihara [13]. The proposed control could maintain the periodic rotation in a certain range of delay with sufficient low energy consumption. The experimentally obtained results indicate that the frequency synchronization overcomes the mistuned difference of delay in the control method.

An extended experimental operation was carried out in Lenci et al. [14] intending to obtain the conditions for rotations of the pendulum excited by waves. The rotational behavior was found for different values of amplitude and frequency, showing robustness in the parameter space. The same robustness could be associated to initial conditions of position and velocity of the pendulum. In general, the experimental results were considered a preliminary proof of the concept presented in $[4,5]$ is practical feasible.

In Litak et al. [15] dynamic responses of the parametric pendulum were obtained experimentally. Recurrence plots technique was performed with time histories in order to find out the kind of motion using only a few cycles. The method based on recurrence quantification analysis (RQA) was able to differentiate oscillations, rotations and the transient chaotic motions.

In Alevras et al. [16] it was analyzed the dynamics of two pendulums on a block linked to a base with a damper and a spring. The base is excited by a sinusoidal force, thus provoking a stochastic parametric excitation of the pendulums. The system mounted intended to explore the idea of extracting energy from sea waves. The numerical analysis focused on the establishment of rotation and in the synchronization.

Yang [17] presented a self-learning terminal sliding-mode control for the synchronization and antisynchronization between two chaotic rotating pendulums with different periods of harmonic parametric excitation. The conditions for stable synchronizations are executed through the Lyapunov stability theorem.

De Paula et al. [18] applied the extended timedelayed control method in order to maintain stable rotational solutions in a pendulum-shaker control system. The method is based on stabilizing some unstable periodic orbits in the chaotic attractor. Afterwards, the period doubling bifurcation is prevented in order to 
(a)

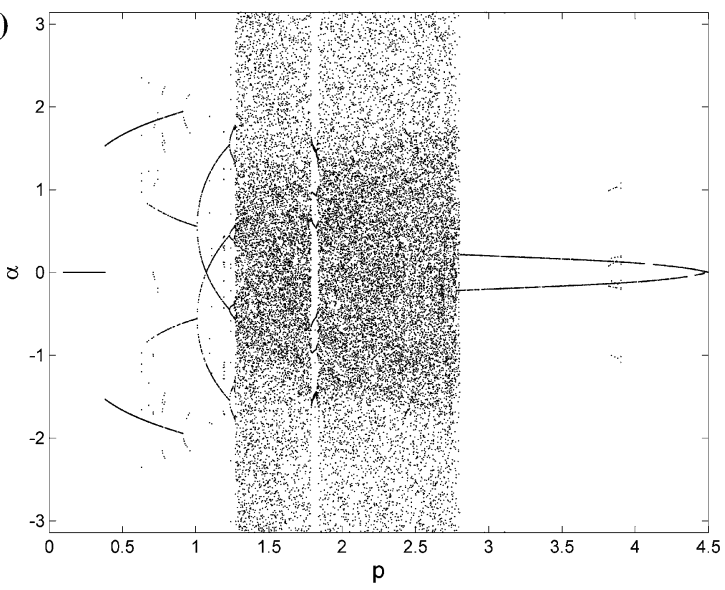

(b)

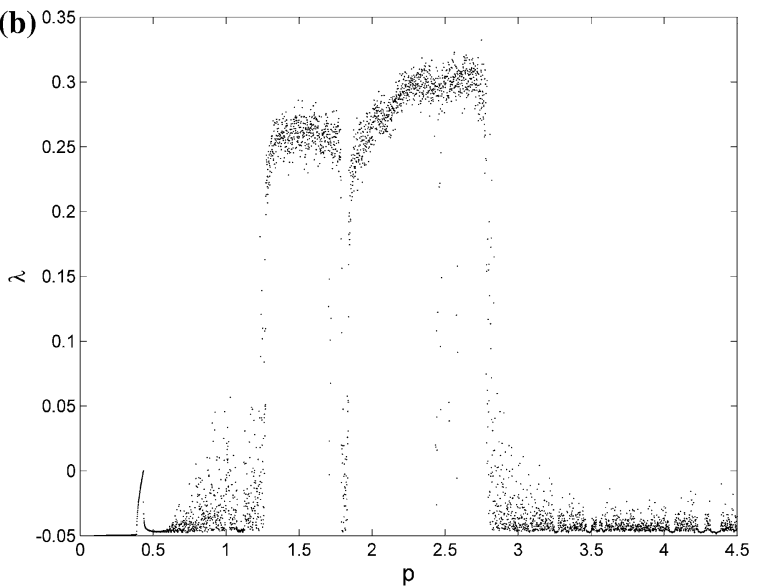

Fig. 4 Graphics for $\omega=1.8$ and $\varepsilon=0$. a Bifurcation diagram, $\mathbf{b}$ Lyapunov exponents

(a)

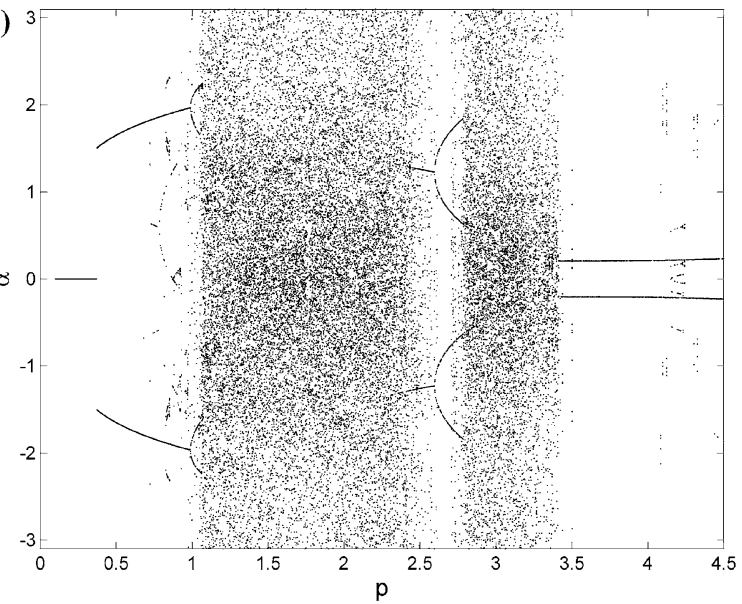

(b)

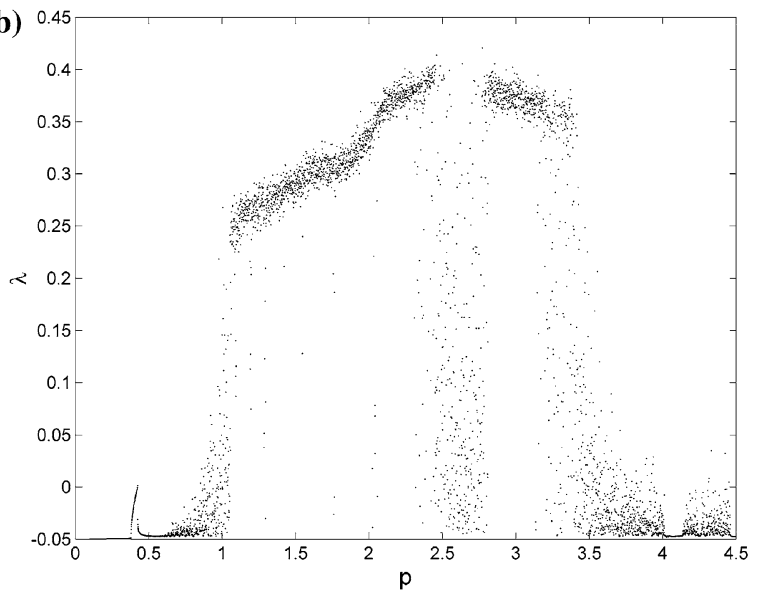

Fig. 5 Graphics for $\omega=1.8$ and $\varepsilon=0.4$. a Bifurcation diagram, b Lyapunov exponents

extend the frequency range where there is a period-1 rotating orbit.

Szemplinska-Stupnicka and Tyrkiel [19] studied the twin-well oscillator, the parametric pendulum and the pendulum driven by an external harmonic force. Chaos is preceded by two and only two asymmetric periodic attractors, where they are simultaneously annihilated through the period-doubling-crisis. It was concluded that the appearance of chaos is not necessarily related to the escape from potential well. It would be a combination of crossing the potential barrier and an oscillatory component. In [19] authors divided chaotic motions in different kinds: oscillating chaos, rotating chaos and tumbling chaos, in which the tumbling chaos involves oscillations and rotations at same time.

Warminski et al. [20] analyzed a non-ideal parametrically and self-exciting model by numerical and analytical methods. The authors verified an interaction between parametric and external excitation which leads to a synchronization. In a comparison, the ideal model exhibited a sort of internal loop in the region of main parametric resonance, while the synchronization had been obtained in the non-ideal model.

In Lenci and Rega [21] it was explored the idea of dynamical integrity. The concept of safe basin is reviewed. The method is based on drawing the basin of attraction as a function of a varying parameter, in a 
(a)

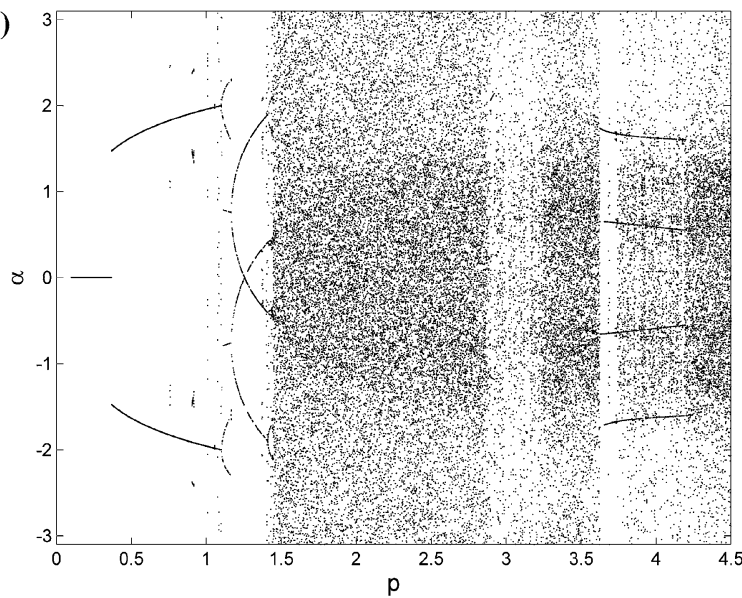

(b)

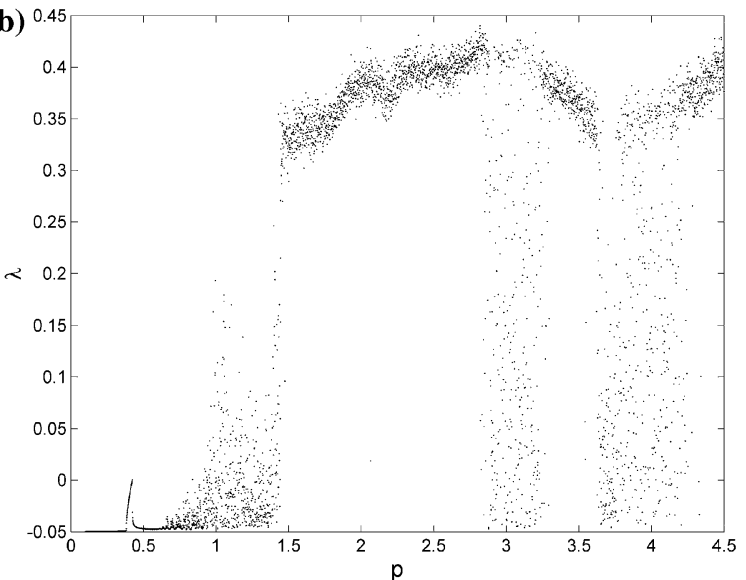

Fig. 6 Graphics for $\omega=1.8$ and $\varepsilon=0.9$. a Bifurcation diagram, $\mathbf{b}$ Lyapunov exponents

(a)

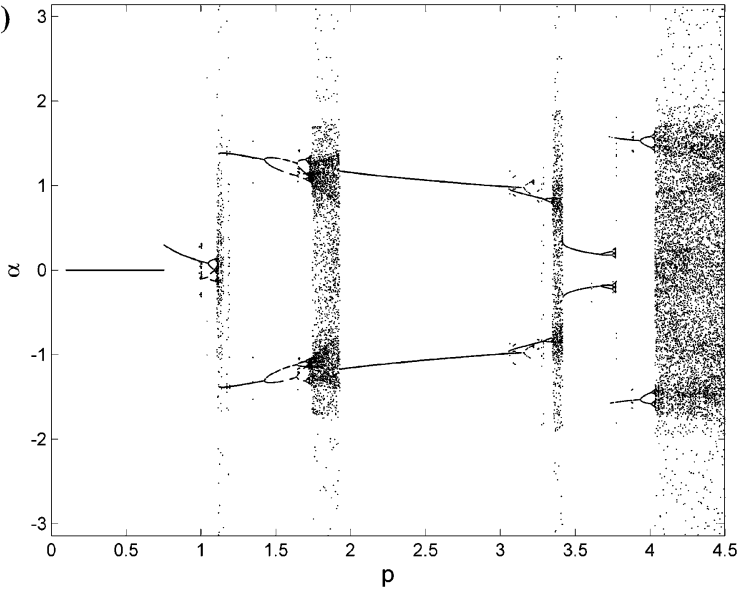

(b)

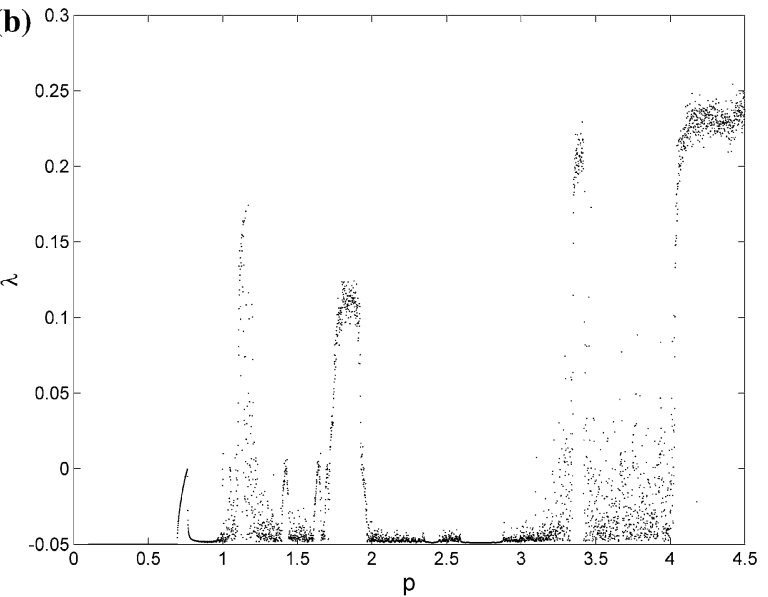

Fig. 7 Graphics for $\omega=0.9$ and $\varepsilon=0$. a Bifurcation diagram, $\mathbf{b}$ Lyapunov exponents

way that one could see "erosion profiles". Considering the dynamical integrity decreases while amplitude of excitation increases. A control method was applied with the purpose to shift the erosion toward larger excitation amplitudes.

In Belato et al. [22, 23] a non-ideal pendular model was analyzed. The mechanism was a crank-shaftslider, which excited horizontally the pendulum support. The crank was powered by an electric motor supplying energy to the pendulum. In [22] it was observed that near the fundamental resonance the pendulum exhibited multi-periodic, quasi-periodic and chaotic motion. The loss of stability occurred after a saddle-node bifurcation, when the pendulum approaches to the angle $\pi / 2$. In [23] it was found that in the low frequencies the system presented few basins of attraction with solutions of greater amplitude, chaotic or multi-periodic. Also, the system presented a boundary-crisis-bifurcation with sequences of interruptions.

Hsieh and Shaw [24] analyzed the dynamic stability of a crank-shaft-slider mechanism where the shaft is considered flexible and the crank is rigid and rotates with constant speed. The results focused on the nonlinear effects which arise from the finite deformations in the rod. An analytical approach is carried out using the method of multiple scales.

An experimental analysis of the crank-shaft-slider mechanism is developed in Halbig and Beale [25]. The purpose was to analyze the behavior of a flexible shaft 
(a)

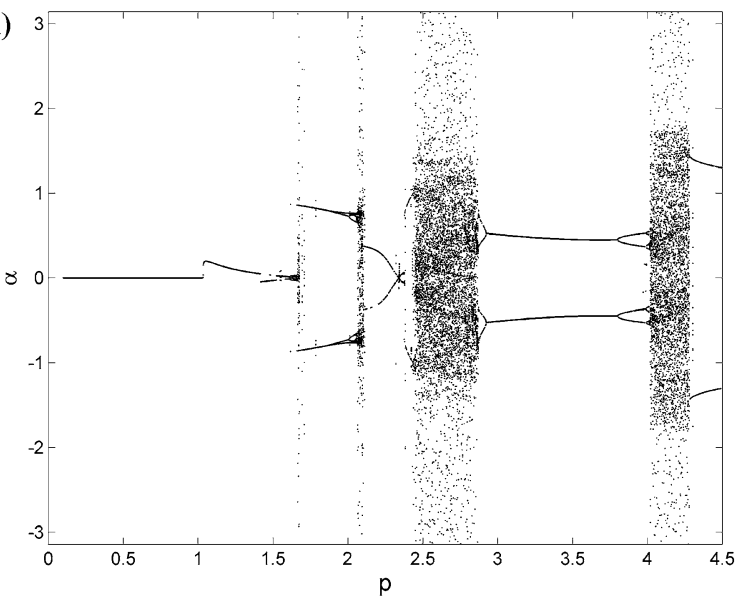

(b)

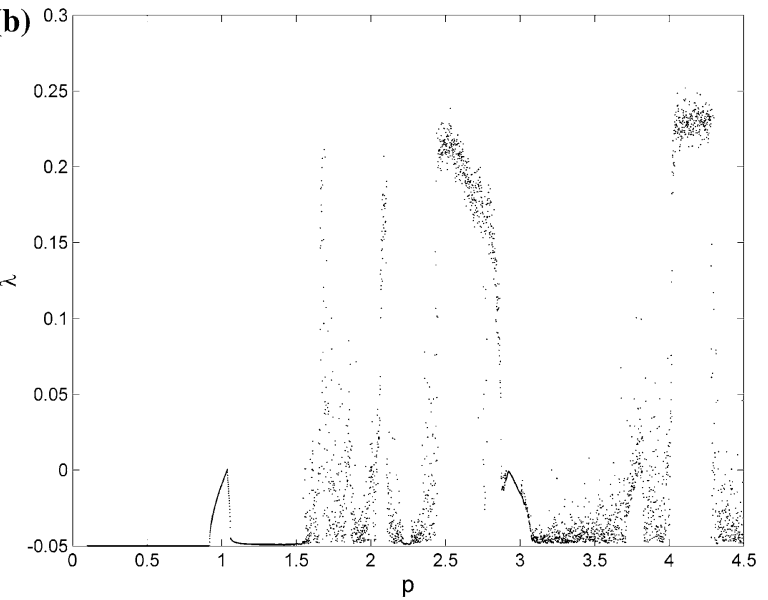

Fig. 8 Graphics for $\omega=0.9$ and $\varepsilon=0.4$. a Bifurcation diagram, b Lyapunov exponents

(a)

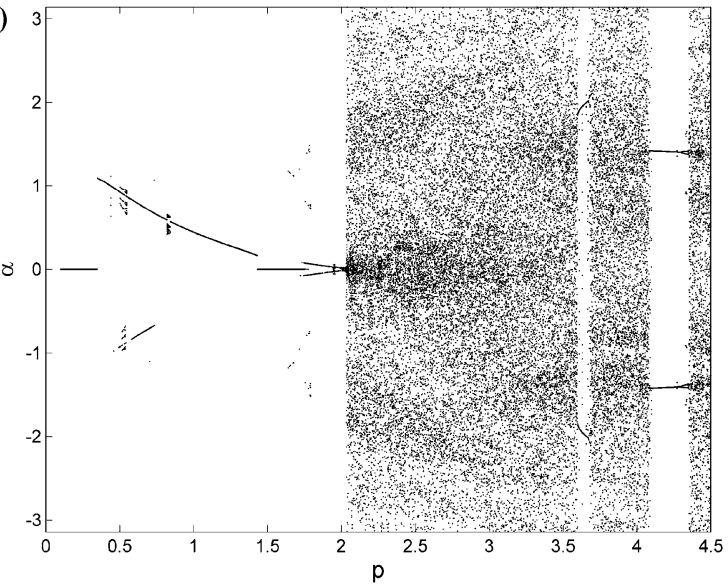

(b)

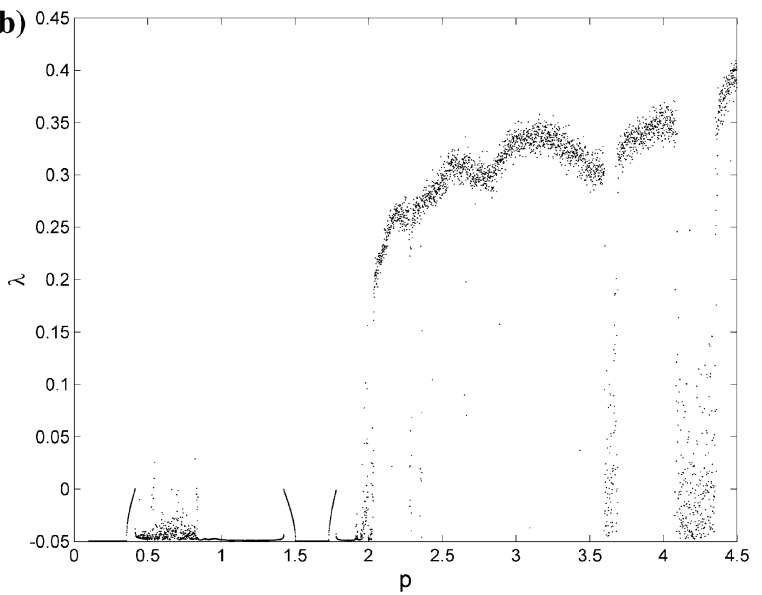

Fig. 9 Graphics for $\omega=0.9$ and $\varepsilon=0.9$. a Bifurcation diagram, b Lyapunov exponents

in a steady state of high speedy crank. The bending of the shaft was studied for different values of the speed and the radius of the crank. The results obtained demonstrated a period doubling and response amplification attributed to the rod flexibility.

In Wauer and Bührle [26] the same mechanism with a flexible rod was analyzed with a non-ideal source of energy. The speedy of crank is no more constant. A DC motor is responsible for the source of energy which is dissipated by the flexible shaft. The results pointed that the fluctuations may be prevented through an appropriate choice of the motor and the system. Transient startup and rundown are also considered in the numerical simulation. Especial attention was given to the influence of flexibility in the dynamic response of system.
In Goudas et al. [27] the crank-shaft-slider mechanism is also under a non-ideal forcing. The crank is supported by compliant bearings. The bearings are considered firstly with linear stiffness, nonlinear stiffness and, finally, hydrodynamics bearings. The mechanism is also compared in dynamics with constant speed of the crank and with a non-ideal source of energy. In the obtained results, the crank-to-rod length ratio demonstrated impact in the dynamic of the system, while the moment of inertia had practically no effect.

In the mathematical model developed for the present paper, the crank-shaft-slider mechanism is analyzed as an extension of the classical parametric pendulum presented in [1-4]. The length of crank 
(a)

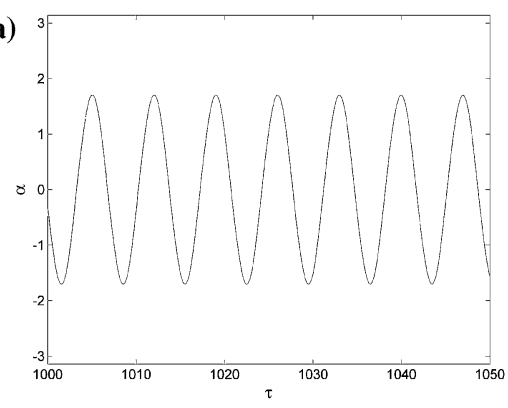

(b)

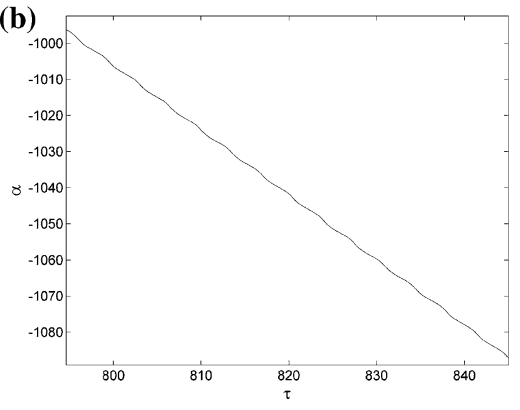

(c)

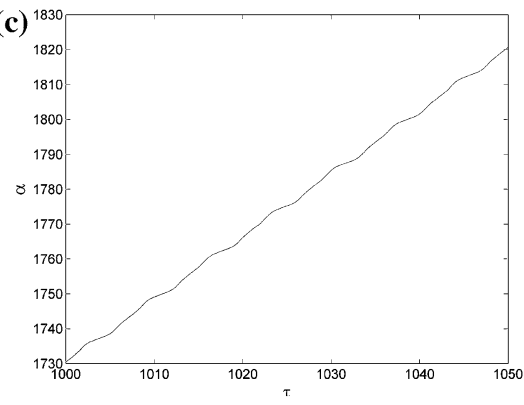

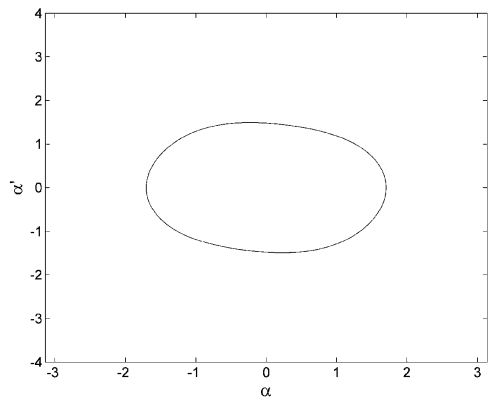
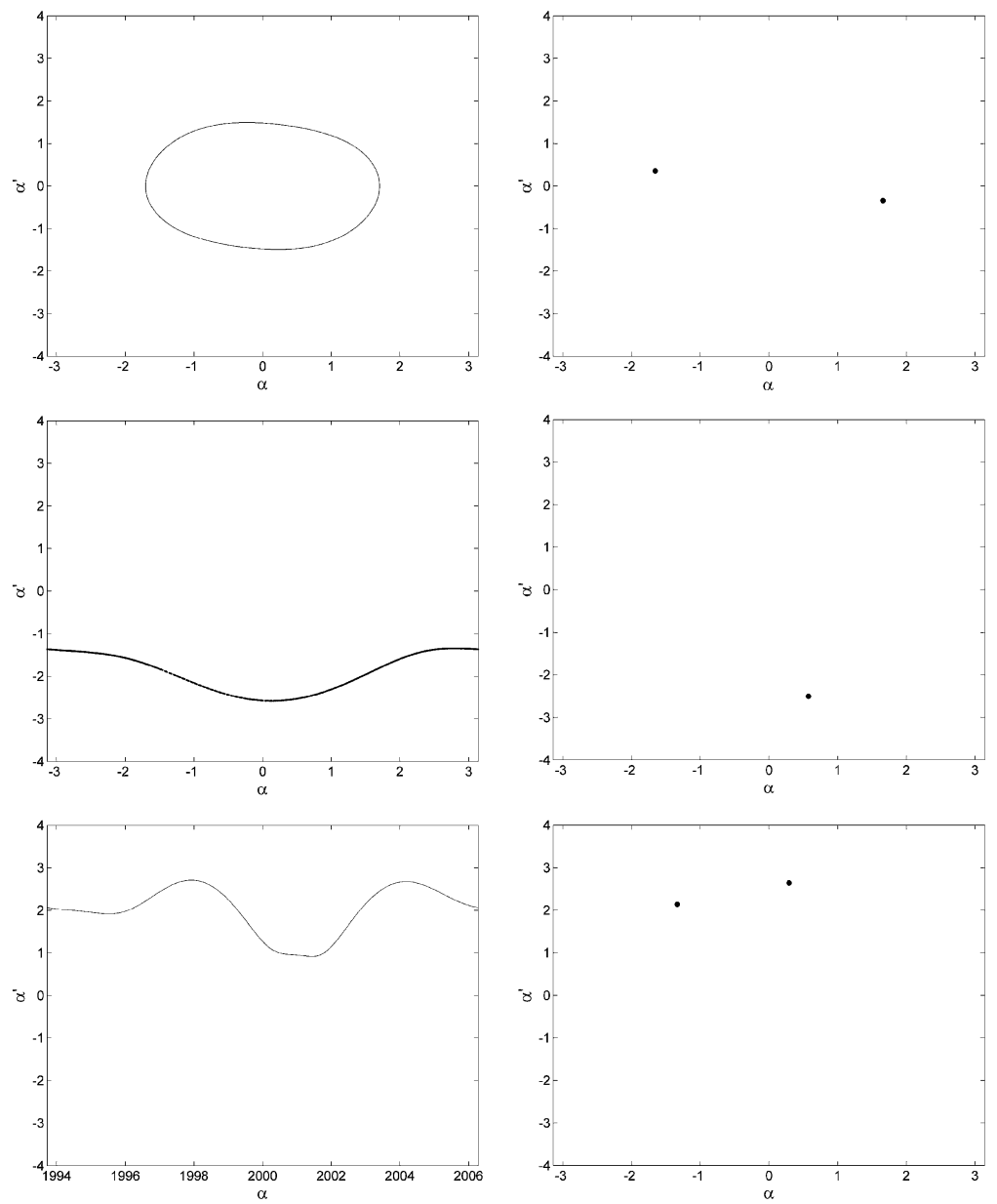

Fig. 10 Time history, phase portrait and Poincaré section for $\omega=1.8$ and $\varepsilon=0$. a $p=0.5, \mathbf{b} p=0.95, \mathbf{c} p=1.15, \mathbf{d} p=1.5$, $\mathbf{e} p=2.8$

divided by the length of the shaft appears at the final formulae as a perturbation on the classical dynamic equation of the parametric excited pendulum used in the literature.

The modeling of the system analyzed here is described in the article followed by an analysis for different dimensionless parameters. Some differences in the pendulum dynamics are observed for the comparison between the classical parametric excitation and due to the crank-shaft-slider.

\subsection{Vibrating system description and governing equations of motions}

The mechanism investigated, here, consists of a rigid weightless rod of length $l$ with a mass $m$ in the end vertically excited by a crank-slider mechanism. The link $a$ is also a weightless rod which rotates with a constant angular velocity whilst the pendulum joint goes up and down moved by the link $b$. In brief, the unique mass considered is the bob at the extreme of the pendulum. An illustration is represented in Fig. 1.

The unique degree of freedom is given by the angle of the pendulum $\alpha$. To write down the Lagrangian function of the mechanism, the position of the mass $m$ was written in terms of the coordinates $\alpha, \theta$ and $\varphi$ in Eq. (1):

$$
\begin{aligned}
& x_{p}=l \sin \alpha \\
& y_{p}=-a \cos \theta-b \cos \varphi-l \cos \alpha
\end{aligned}
$$

Taking into account the trigonometric relation between $\theta$ and $\varphi$ we may substitute in Eq. (1) what 
(d)

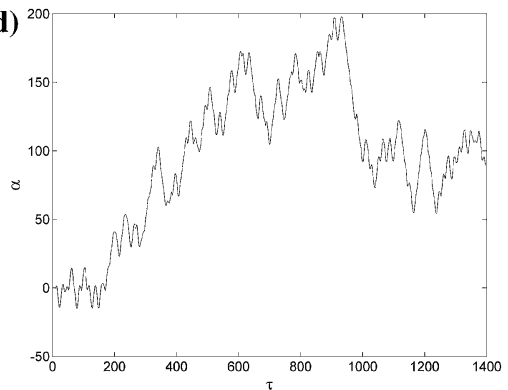

(e)

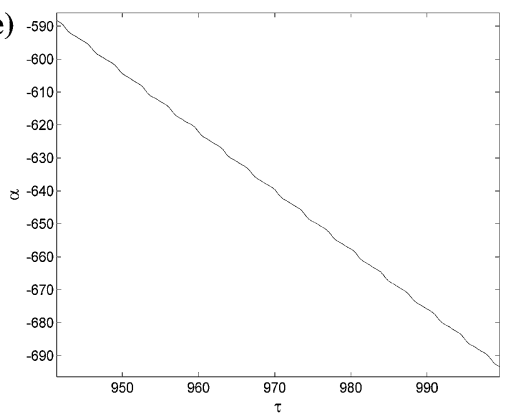

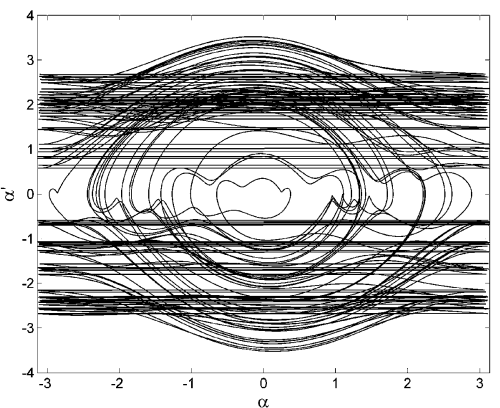
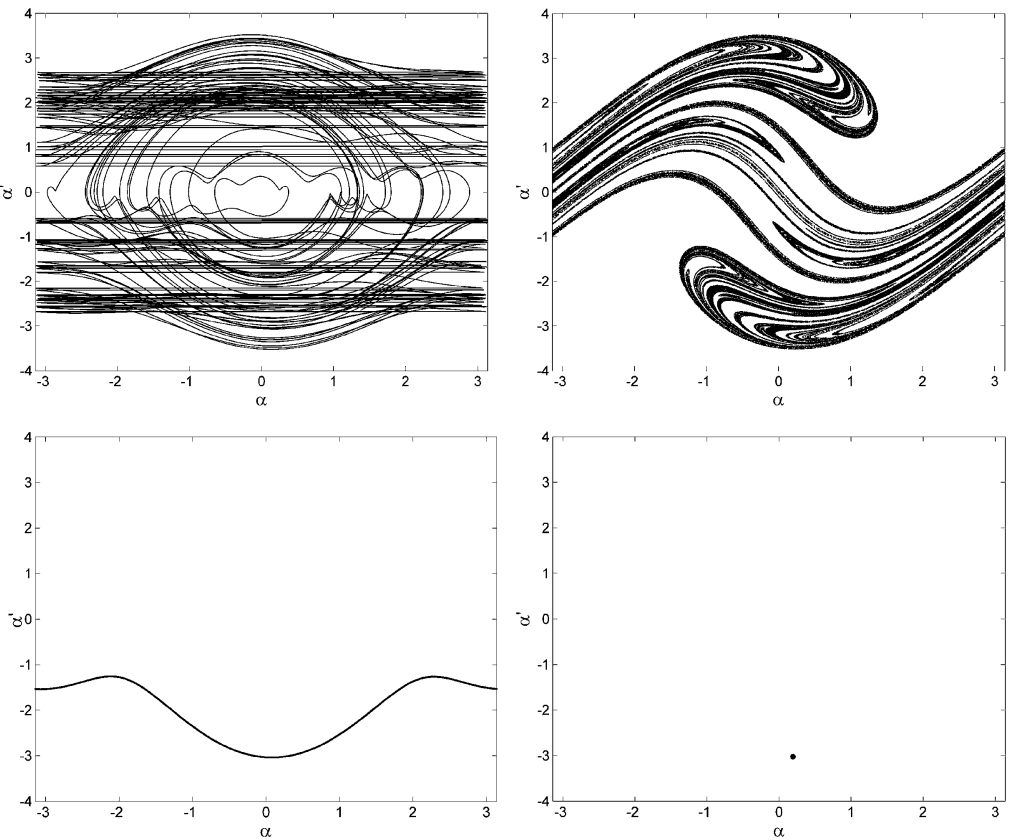

Fig. 10 continued

is written in terms of $\varphi$. The value of $\cos \varphi$ will be substituted by the right side of Eq. (2):

$\cos \varphi=\left(1-\frac{a^{2}}{b^{2}} \sin ^{2}(\theta)\right)^{1 / 2}$

Derivation with respect to the time $t$, thus the velocities are given by Eq. (3):

$\dot{x}_{p}=l \dot{\alpha} \cos \alpha$

$\dot{y}_{p}=a \dot{\theta} \sin \theta+\frac{a^{2} \dot{\theta} \sin \theta \cos \theta}{b\left(1-\frac{a^{2}}{b^{2}} \sin ^{2} \theta\right)^{1 / 2}}+l \dot{\alpha} \sin \alpha$

Introducing a dimensionless variable $F$ in Eq. (4), dependent on $\theta$ and its respective derivative, similarly to Belato et al. [22]:

$$
\begin{gathered}
F=\sin \theta+\frac{a \sin \theta \cos \theta}{b\left(1-\frac{a^{2}}{b^{2}} \sin ^{2} \theta\right)^{1 / 2}} \\
\dot{F}=\dot{\theta} \cos \theta+\frac{a \dot{\theta} \cos (2 \theta)}{b\left(1-\frac{a^{2}}{b^{2}} \sin ^{2} \theta\right)^{1 / 2}} \\
+\frac{a^{3} \dot{\theta} \sin ^{2}(2 \theta)}{4 b^{3}\left(1-\frac{a^{2}}{b^{2}} \sin ^{2} \theta\right)^{3 / 2}}
\end{gathered}
$$

Therefore the velocities in Eq. (3) are rewritten in Eq. (5) employing the $F$ function: $\dot{x}_{p}=l \dot{\alpha} \cos \alpha$

$\dot{y}_{p}=a \dot{\theta} F+l \dot{\alpha} \sin \alpha$

The kinetic energy $T$ and the potential energy $V$ in form of $x_{p}$ and $y_{p}$ coordinates are written in Eqs. (6) and (7):

$T=\frac{1}{2} m\left(\dot{x}_{p}^{2}+\dot{y}_{p}^{2}\right)$

$V=m g y_{p}$

The kinetic energy $T$ and the potential energy $V$ of the examined system in terms of $\theta$ and $\alpha$ coordinates are written, respectively, in the Eqs. (8) and (9):

$T=\frac{1}{2} m\left[l^{2} \dot{\alpha}^{2} \cos ^{2} \alpha+(a \dot{\theta} F+l \dot{\alpha} \sin \alpha)^{2}\right]$

$V=-m g l \cos \alpha$

$$
-m g b\left(1-\frac{a^{2}}{b^{2}} \sin ^{2} \theta\right)^{1 / 2}-m g a \cos \theta
$$

Through the prior forms of kinetic and potential energy we apply Lagrange's equation with respect to the coordinate $\alpha$ :

$\frac{d}{d t} \frac{\partial L}{\partial \dot{\alpha}}-\frac{\partial L}{\partial \alpha}=G_{\alpha}^{N C}$ 

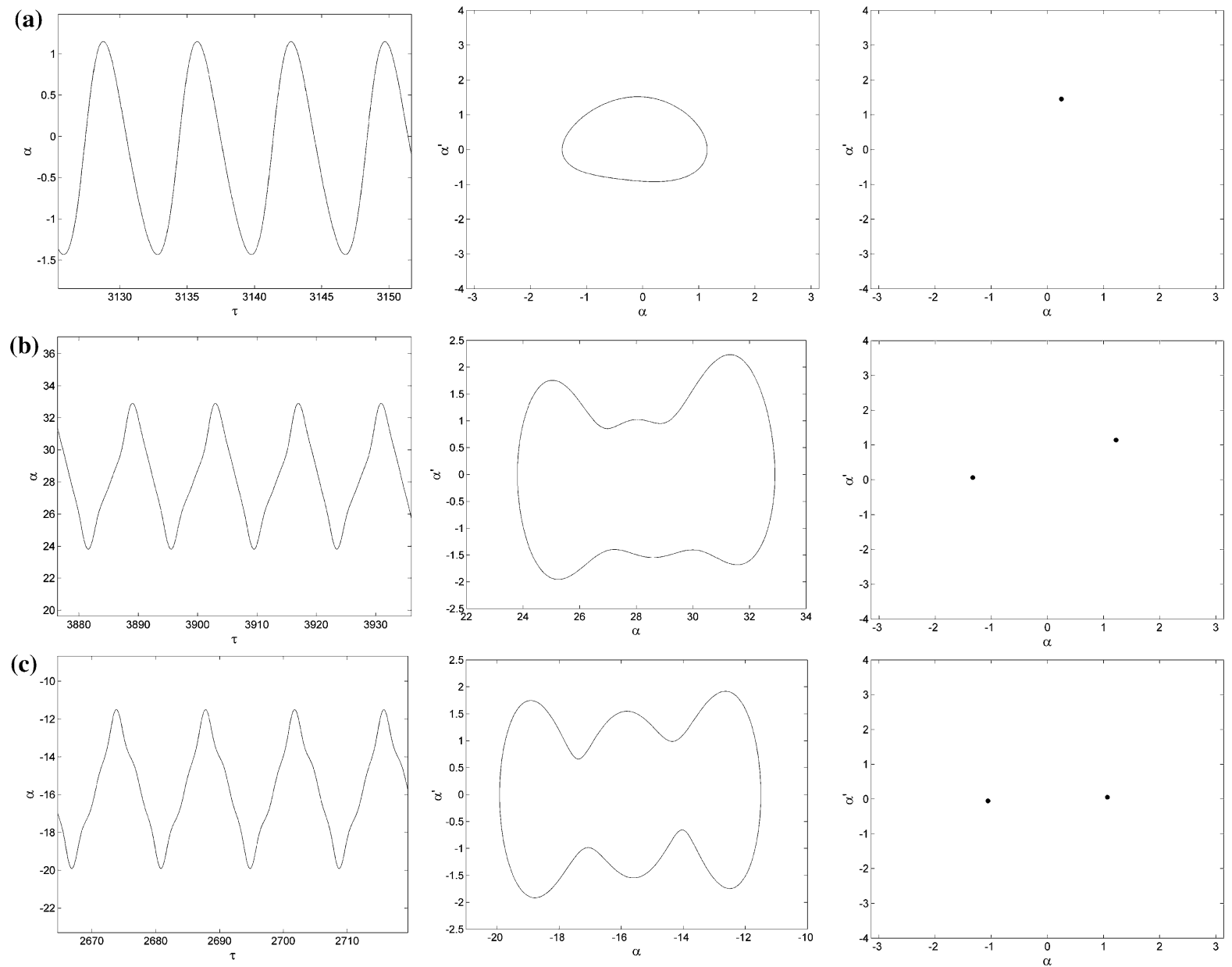

Fig. 11 Time history, phase portrait and Poincaré section for $\omega=0.9$ and $\varepsilon=0$. a $p=0.8, \mathbf{b} p=1.5$, c $p=2.5$, d $p=3.6$, e $p=4.4$

The term in the right-hand side of Eq. (10) stands for the nonconservative force present in the motion. This force opposes to the motion of the pendulum in direction $\alpha$ and might be due to the friction in the joint which supports the pendulum or can be due to the viscosity of the medium. The friction opposing to the movement of the slider was not considered in the modeling because its motion is determined by the velocity and geometry of the crank and the shaft. The friction coefficient is represented by letter $c$ and the generalized force in Eq. (11) has dimension of torque.

$G_{\alpha}^{N C}=-c l^{2} \dot{\alpha}$

Calculating the derivatives in Eq. (10) the resultant differential equation is in Eq. (12):

$m l^{2} \ddot{\alpha}+m a l \dot{\theta} \dot{F} \sin \alpha+m g l \sin \alpha=-c l^{2} \dot{\alpha}$
Dimensionless time $\tau$ is inserted through terms introduced in Eq. (13):

$$
\begin{aligned}
\omega_{0} & =\sqrt{g / l} \\
\tau & =\omega_{0} t \\
\frac{d F}{d \tau} & =\frac{1}{\omega_{0}} \frac{d F}{d t} \\
\frac{d \alpha}{d \tau} & =\frac{1}{\omega_{0}} \frac{d \alpha}{d t} \\
\frac{d^{2} \alpha}{d \tau^{2}} & =\frac{1}{\omega_{0}^{2}} \frac{d^{2} \alpha}{d t^{2}} \\
\frac{d \theta}{d \tau} & =\frac{1}{\omega_{0}} \frac{d \theta}{d t}=\omega
\end{aligned}
$$

From here on, the derivatives with respect to time $t$ will be represented with a dot on the symbol, while 

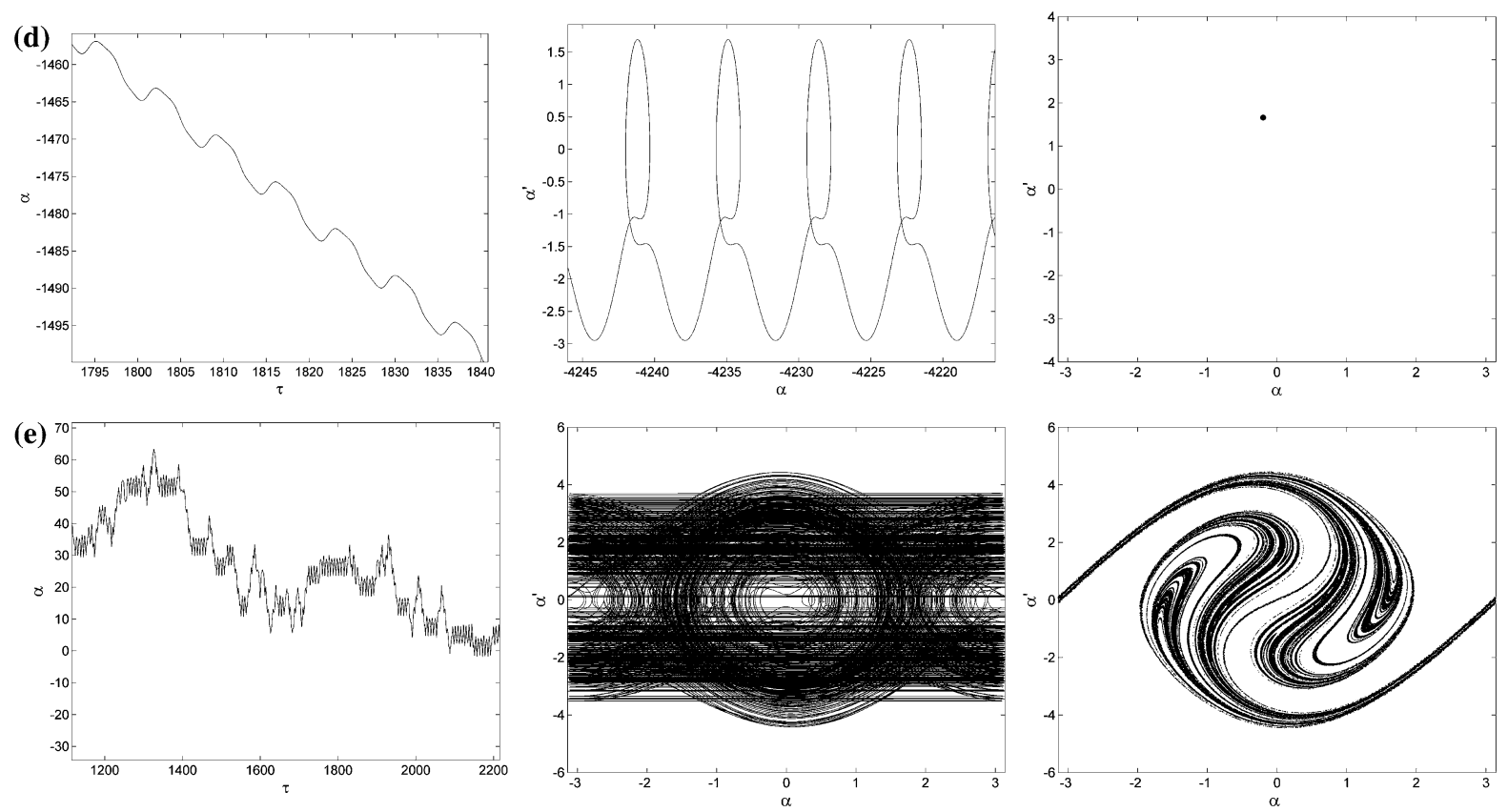

Fig. 11 continued

the apostrophe means a derivative with respect to the dimensionless time $\tau$.

Substituting in Eq. (12) the terms with derivatives with respect to $t$ for terms with derivatives with respect to $\tau$ and afterwards dividing the whole equation for $m l \omega_{0}^{2}$, the dimensionless equation will be obtained:

$\alpha^{\prime \prime}+\frac{c}{m \omega_{0}} \alpha^{\prime}+\sin \alpha=-\frac{a}{l} \omega F^{\prime} \sin \alpha$

The Eq. (14) turns into the classical parametric pendulum equation [1-4] when the ratio $a$ over $b$ is negligible. That will become explicit in the next steps.

Dimensionless parameters need to be inserted to give a better form and allow an appropriate comparison with the classical parametrically-excited pendulum in the manner it is analyzed in literature. These parameters are in Eq. (15):

$p=\frac{a}{l} \omega^{2}$

$\varepsilon=\frac{a}{b}$

$\gamma=\frac{c}{m \omega_{0}}$

When the parameter $\varepsilon$ equals zero, the dimensionless Eqs. (14) and (16) turns into the equation found in [1-4]. It also means that when the maximum angle of $\varphi$ approaches zero, the excitation by the crank-shaftslider becomes a harmonic excitation similarly to the classical parametrically excited pendulum. However, if the value of $\varepsilon$ is not negligible, the final dynamic equation for this mechanism is exactly represented by the Eq. (16):

$$
\begin{aligned}
& \alpha^{\prime \prime}+\gamma \alpha^{\prime}+\sin \alpha[1+p \cos (\omega \tau) \\
& \left.+\varepsilon \frac{p \cos (2 \omega \tau)}{\left(1-\varepsilon^{2} \sin ^{2}(\omega \tau)\right)^{1 / 2}}+\varepsilon^{3} \frac{p \sin ^{2}(2 \omega \tau)}{4\left(1-\varepsilon^{2} \sin ^{2}(\omega \tau)\right)^{3 / 2}}\right] \\
& \quad=0
\end{aligned}
$$

\section{Numerical results}

\subsection{Basins of attractions}

For each attractor in a dynamical system, there is a set of initial conditions leading to it. This set of initial conditions is called the basin of attraction of a determined attractor. In the case of the parametric pendulum, those initial conditions are the initial angular position and the initial velocity of the 
(a)

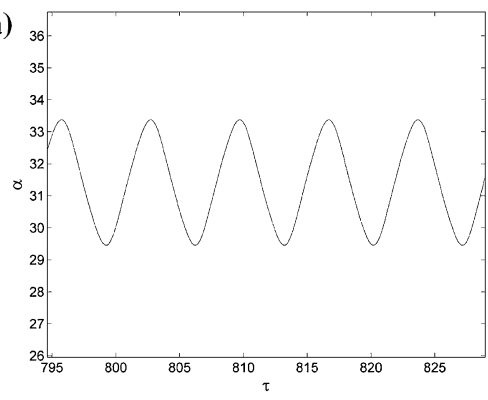

(b)

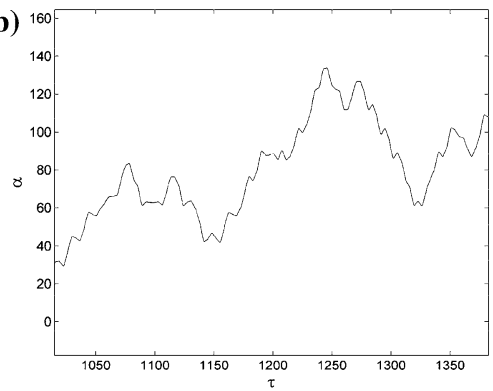

(c)

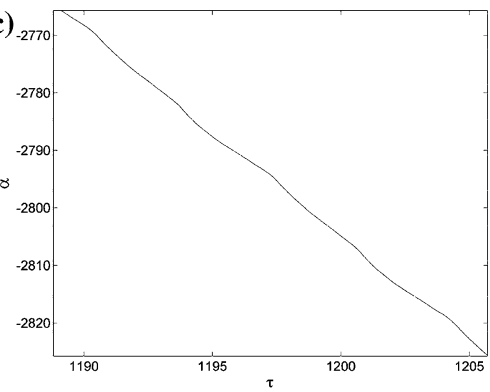

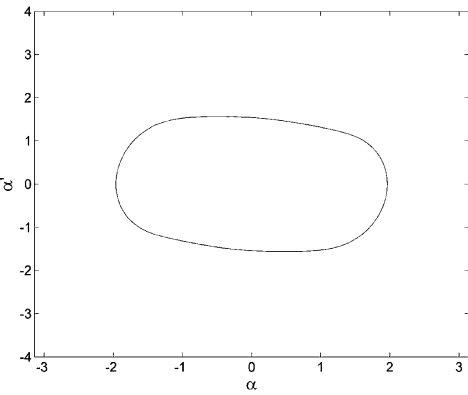
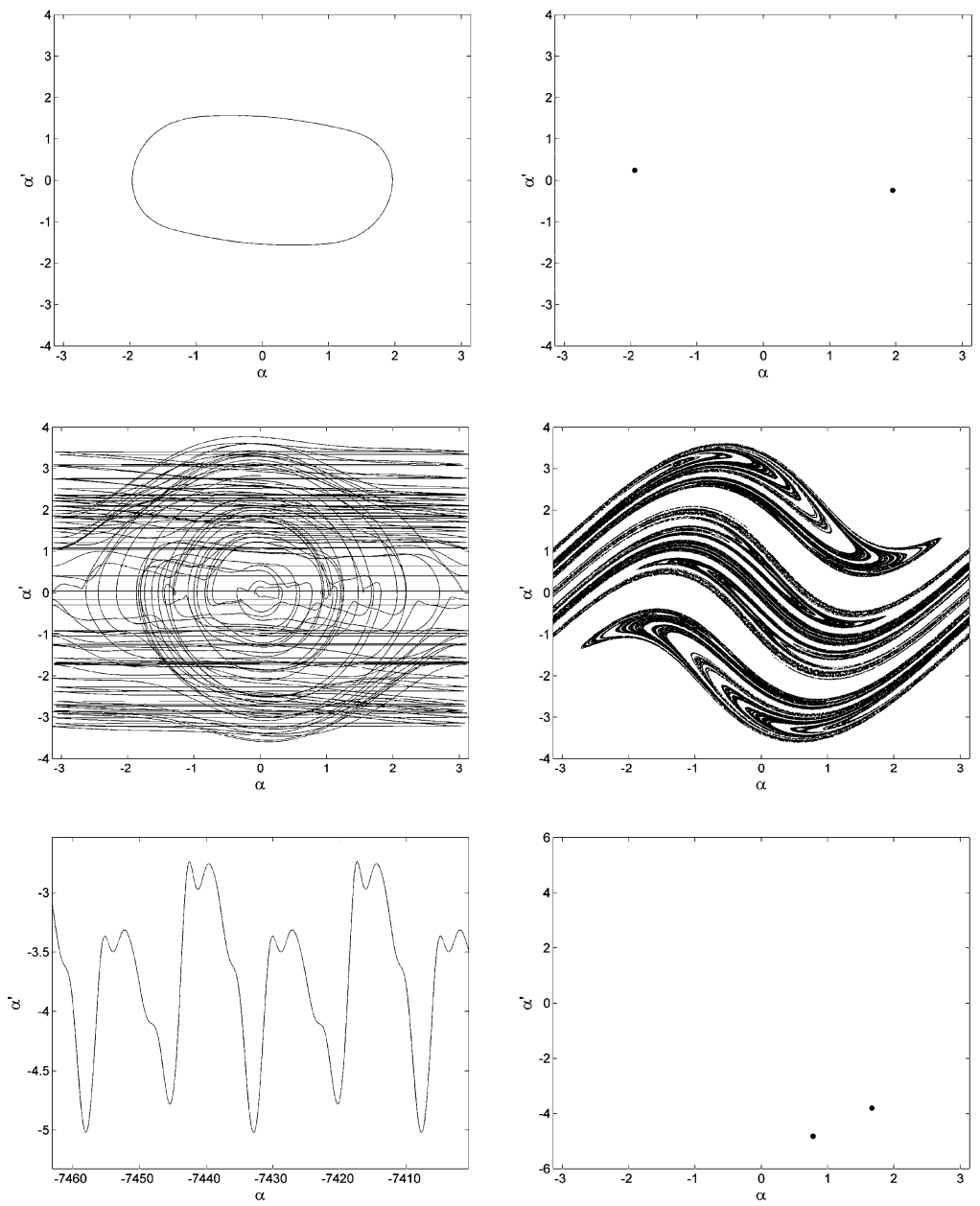

Fig. 12 Time history, phase portrait and Poincaré section for $\omega=1.8$ and $\varepsilon=0.4$. a $p=0.95, \mathbf{b} p=1.8, \mathbf{c} p=2.7$, d $p=2.8$, $\mathbf{e} p=3.5$

pendulum. Basins of attractions are useful to show how different values in initial conditions lead to different kinds of motion. An analysis considering basins of attraction for the parametric pendulum is developed in $\mathrm{Xu}$ et al. [4].

The building process consists in initially investigate how much time is necessary to obtain a steady state in the dynamics of the pendulum. Afterwards, varying the initial conditions for the position $\alpha$ from $-\pi$ to $\pi$ and the velocity $\alpha^{\prime}$ from -4 to 4 , using the fixed parameters $p=0.5, \omega=1.8$ and $\gamma=0.1$, it was verified that the regime was reached in most of cases in 300 times the excitation period. That was the time elapsed for results plotted in Fig. 2a, b, c. An exception for Fig. 2d, it was necessary 600 times the excitation period to stabilize in $\varepsilon$ equals to 0.9 .
It is more convenient for plotting that the motions are stable, instead of chaotic. In this way, each beginning point of a region is more likely to reach a specific attractor. It means that the regions from different attractors hardly ever will overlap one another. Concerning the method of constructing the basins of attraction, it was used the Poincaré maps to find the attractors. The attractors found in this paper corroborates with $\mathrm{Xu}$ et al. [4] for the case of $\varepsilon$ equals zero. Varying the $\varepsilon$ value, the same attractor considering the kind of motion is found, where the difference was a small displacement.

The attractors are a period- 2 oscillation and two attractors of period-1 rotation. Those rotational attractors are each one to a direction. One rotates to the positive direction and the other to the negative direction. 

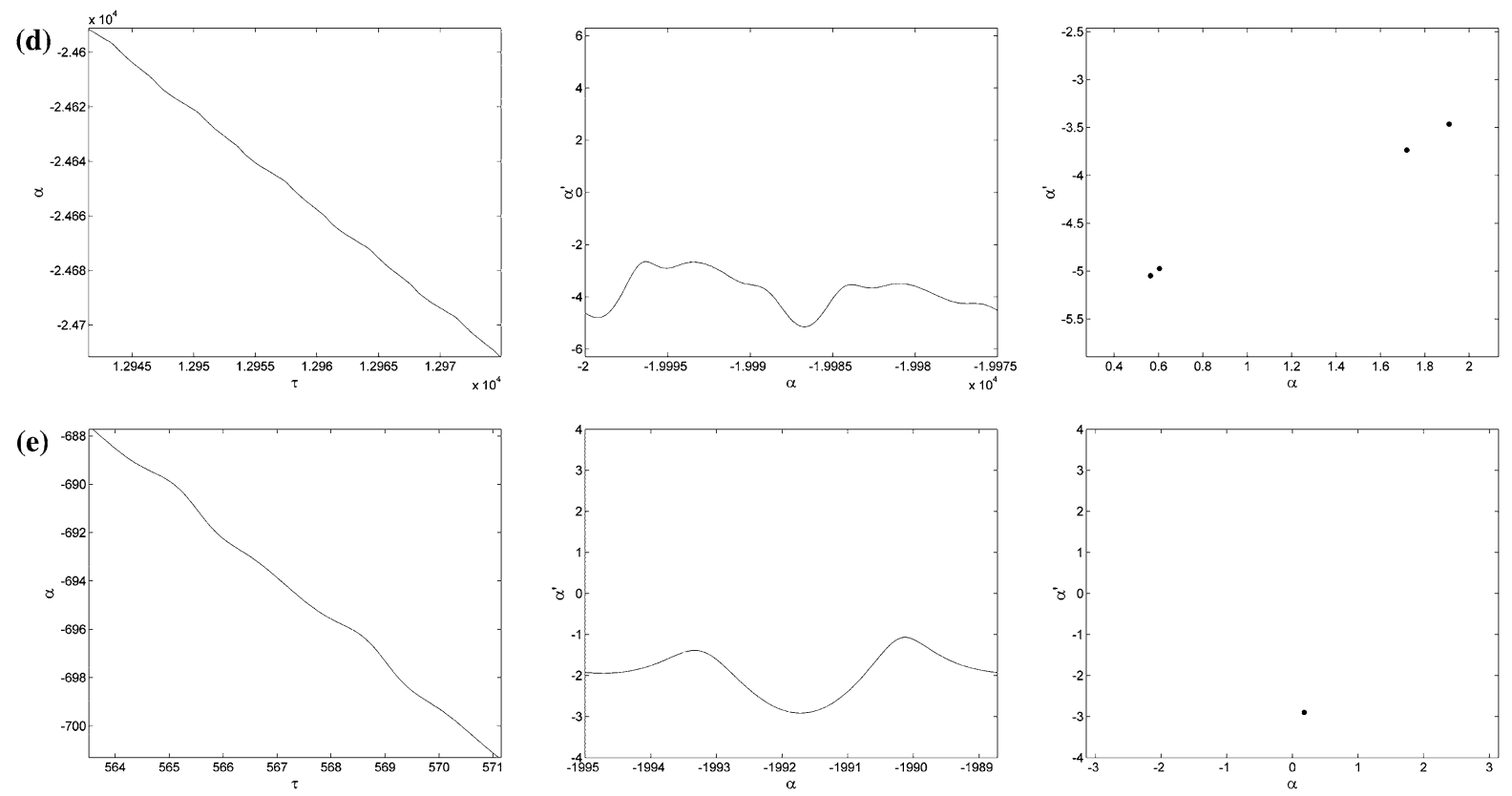

Fig. 12 continued

Afterwards the initial conditions were tested for the interval of $-\pi$ to $\pi$ for position and -4 to 4 for velocity, using a step of 0.01 . The period- 2 oscillating attractors were marked with squares in the graphic and the rotating period-1 with circles. For each picture, there were two period-1 rotating attractors represented in the respective region. The positive rotating region is plotted in black and the negative in red. The region in green is the area of attraction of the period-2 oscillation attractor.

The basins of attractions were built based on some fixed parameters and varying the $\varepsilon$. Using the parameters $p=0.5, \omega=1.8$ and $\gamma=0.1$, it was verified that even varying the value of $\varepsilon$, the attractors in the phase portrait will nearly maintain a constant position as one may see in Table 1 . The attractors were obtained by Poincaré sections. The position for rotating solutions could be found displacing the angular position to the interval between $-\pi$ and $\pi$, considering zero as the rest position. Otherwise, the basin of attraction could not consider rotational solutions.

The method used to integrate the differential equation was the 4th order Runge-Kutta and the time step is the period of $\omega$ divided by 400 . As mentioned, the positions of the attractors obtained demonstrate not varying so much whist varying the $\varepsilon$ parameter.

\subsection{Parameter space plot}

A parameter space plot is a diagram, which represents the distribution of different kinds of motion when two or more parameters of the system are varied. In this study, the analysis was done by observing different bifurcation diagrams for $\omega$ between 0.5 and 3.5 , in every 0.2 or 0.1 when necessary. In each bifurcation diagram the value of $p$ was varied from 0 to 4.5. Any possibility of change of kind of motion was analyzed with Poincaré sections, phase portraits and time histories. Therefore the results could indicate that some regions of kinds of motions might by marked. On the other hand, some regions could not be associated to any type.

The Lyapunov exponents were also worked out intending to aid the parameter plot construction. The numerical method present in Wolf et al. [28] was the algorithm used.

At the three Fig. 3a, b, c the regions were divided in $\mathrm{R} 1$ for period-1 rotation, $\mathrm{R} 2$ for period-2 rotation, $\mathrm{O} 2$ for period-2 oscillation, $\mathrm{C}$ for chaotic result, Or2 for period-2 oscillation-rotation and finally $U$ meaning "uncertain" for the regions where different kinds of motion occurs in narrow zones, hence not allowing to establish a pattern. The results obtained in Fig. 3a are 
(a)

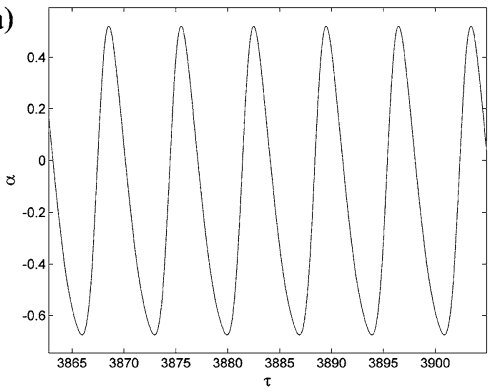

(b)

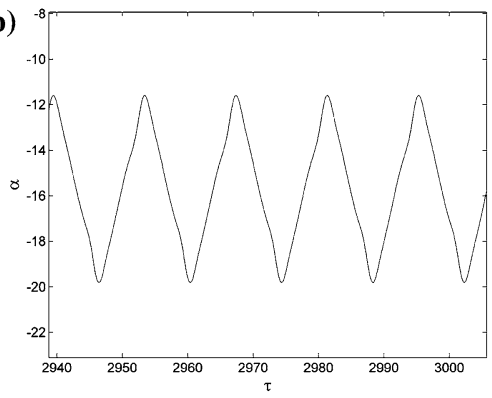

(c)

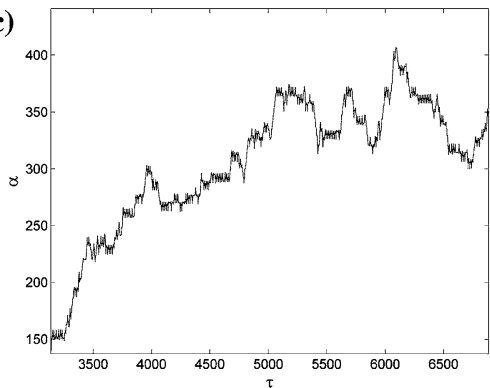

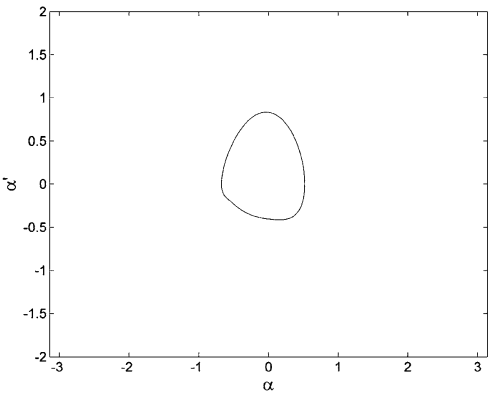
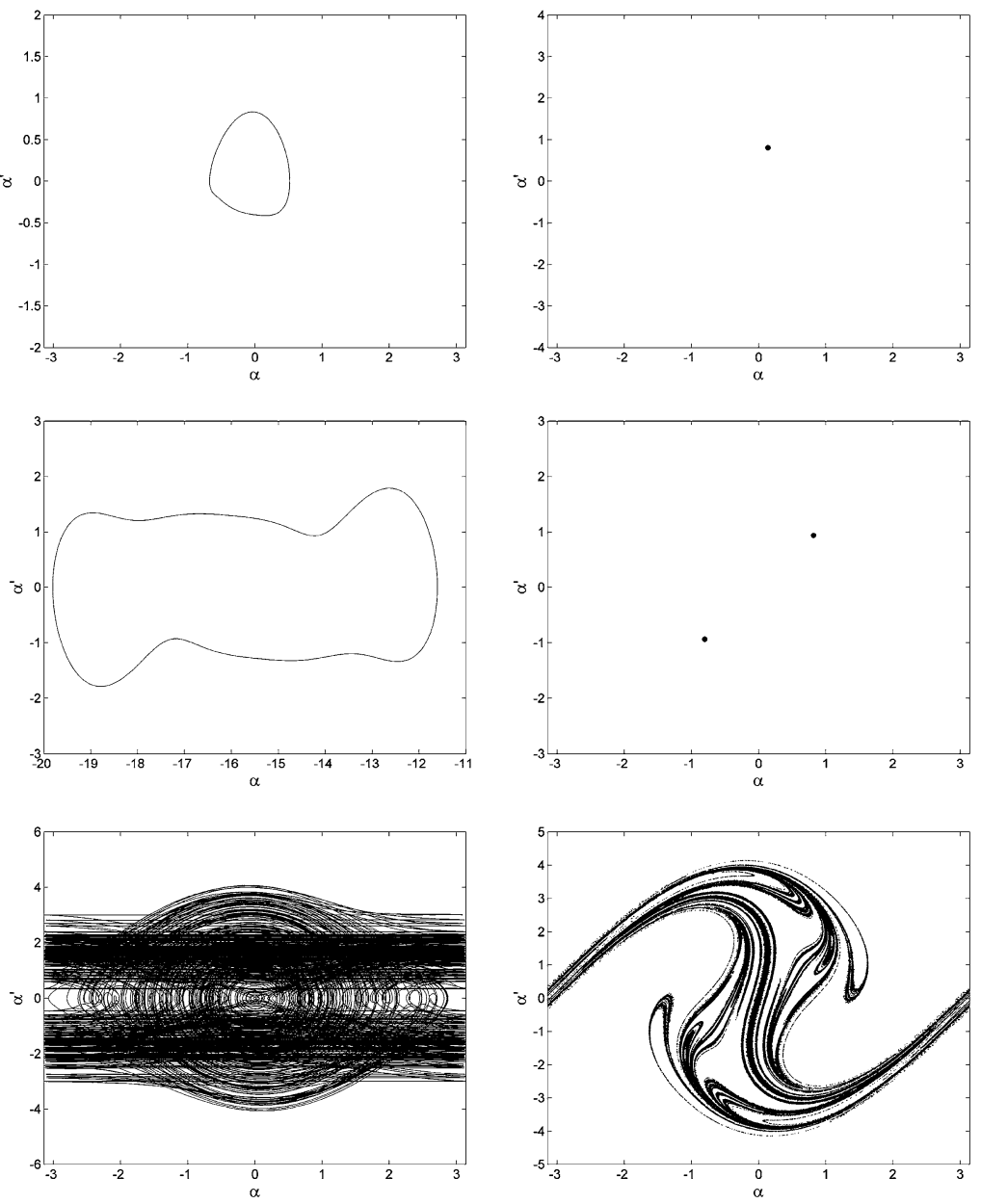

Fig. 13 Time history, phase portrait and Poincaré section for $\omega=0.9$ and $\varepsilon=0.4$. a $p=1.1, \mathbf{b} p=1.8, \mathbf{c} p=2.5$, d $p=3.0$, $\mathbf{e} p=4.5$

very similar to the parameter plot presented in Xu et al. [4], while the Fig. 3b, c are originals (new) and demonstrate the region of uncertain results increases as the parameter $\varepsilon$ increases, when it is considered the presence of the crank-shaft-slider mechanism.

\subsection{Bifurcation diagrams and Lyapunov exponents}

Bifurcation diagrams were used to construct the parameter space plots with many values for $\omega$ from 0.5 to 3.5. Among these diagrams, it is more important to point those correspondents to the resonant regions. According Butikov [29] the resonant regions for the classical parametrically excited pendulum are found in the series $\Omega / \omega_{0}=2 / n$, for $n=1,2,3 \ldots$, where the $\Omega$ is the external exciting frequency and $\omega_{0}$ the natural frequency, that is why it was chosen the values $\omega$ equals 1.8 and 0.9 for a more detailed analysis. Bifurcation diagrams are followed by Lyapunov exponents to ensure the chaotic behavior for some regions. The results for Lyapunov exponents were acquired from the last $10 \%$ of elapsed time in 1000 times the excitation period. For each value of $p$ it was taken the greatest numerical value of Lyapunov exponent $\lambda$ in that last $10 \%$ of the time elapsed. The step used in varying the parameter $p$ was 0.001 , this was enough to represent a good amount of points in Lyapunov exponents graphic as so as in bifurcation diagrams. However, the time executed for bifurcation 
(d)

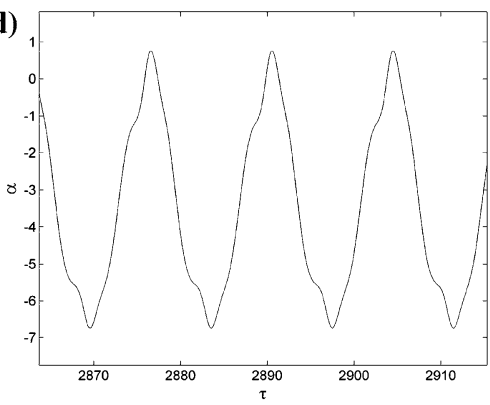

(e)

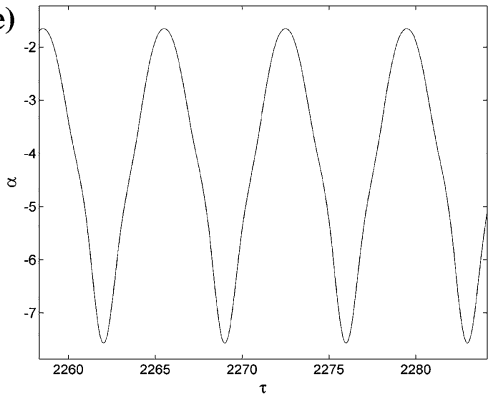

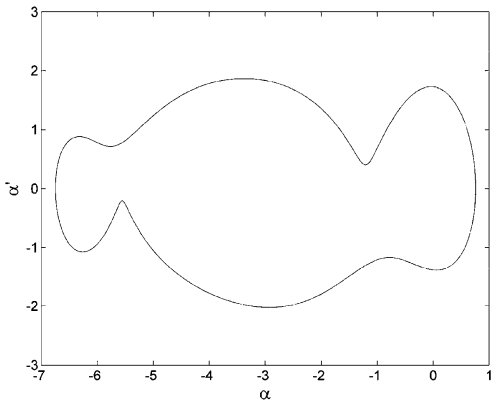
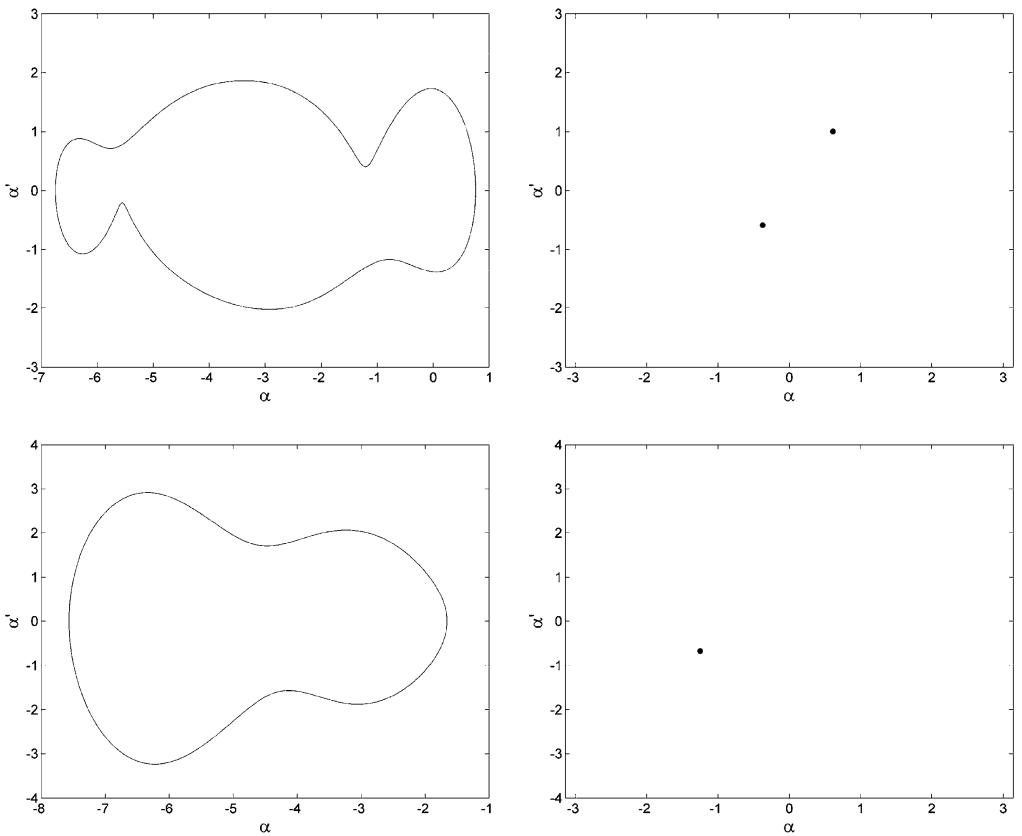

Fig. 13 continued

diagrams was chosen at 300 times the excitation period, because more time, in general, did not exhibit different features in graphics.

\subsubsection{Frequency $\omega=1.8$}

For the parameter $\omega$ equal to 1.8 it was observed an enlargement of the region with chaotic behavior comparing the Figs. 4, 5 and 6. Increasing the value of $\varepsilon$, the chaotic region increases accordingly. In common, the bifurcation show that for higher values of $p$ the pendulum will exhibit rotating solutions, what may also be observed in the parameter space plots.

\subsubsection{Frequency $\omega=0.9$}

For the frequency associated to the parameter $\omega$ equal to 0.9 it was not observed a spread of the chaotic region comparing Figs. 7 and 8. Indeed, it might be seen a change of place for the chaotic region and others kinds of motion. Very narrow regions of chaos are present near $p$ equals to 1.1, 1.8 and 3.4 in Fig. 7, for $\omega=0.9$ and $\varepsilon=0$. In Fig. 8 one can see larger regions of chaos in 2.6 and 4.2, but they are in different places of the chaotic regions in Fig. 7. In Fig. 9 the chaotic regions are considerably larger than in Figs. 7 and 8 , however, they also do not have the same chaotic area.

\subsection{Time histories, phase portraits and Poincaré} sections

The idea in this section is to analyze the kind of motion for each zone in bifurcation diagrams. Therefore it would be necessary the usage of time histories, phase portraits and Poincaré sections. For example, looking at the bifurcation diagram one cannot distinguish a period-1 rotation from a period2 oscillation.

Figure 10 illustrates time histories, phase portraits and Poincaré sections for parameters $\omega=1.8$ and $\varepsilon=0$. In Fig. 10a, for $p$ equals 0.5 , the result is a period-2 oscillation of the pendulum. A period-1 pure rotation was found in Fig. 10b, as well as in Fig. 10e, for $p$ equal to 0.95 and 2.8, respectively. In Fig. 10c, a period-2 pure rotation occurs for $p$ equal to 1.15 and a chaotic attractor is found for $p$ equal to 1.5 , similarly to that in $\mathrm{Xu}$ et al. [4].

Times histories, phase portraits and Poincaré sections for $\omega=0.9$ and $\varepsilon=0$ are represented in Fig. 11 . Period-2 oscillations-rotations occur in Fig. 11b, c. A period-1 oscillation appears for $p$ equal to 0.8 in 
(a)

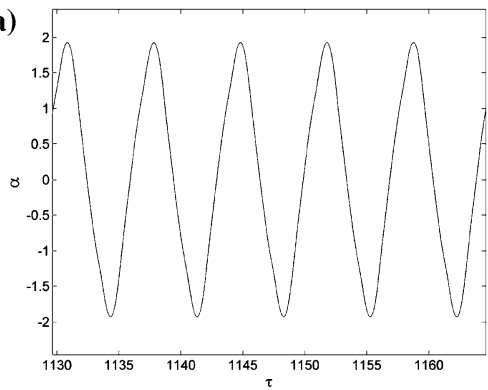

(b)

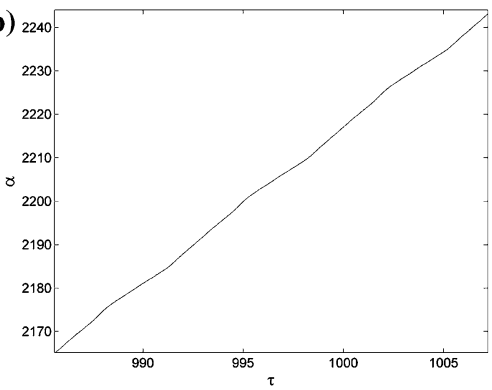

(c)

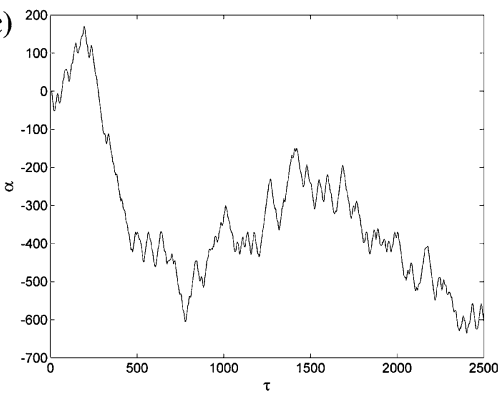

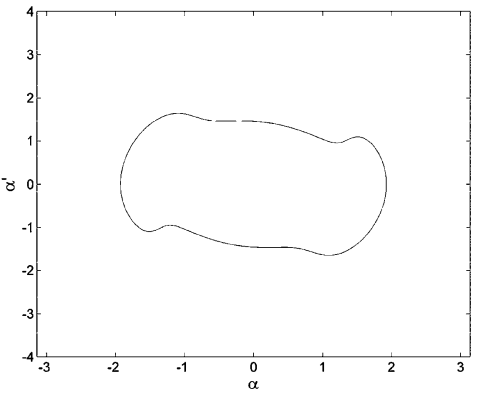
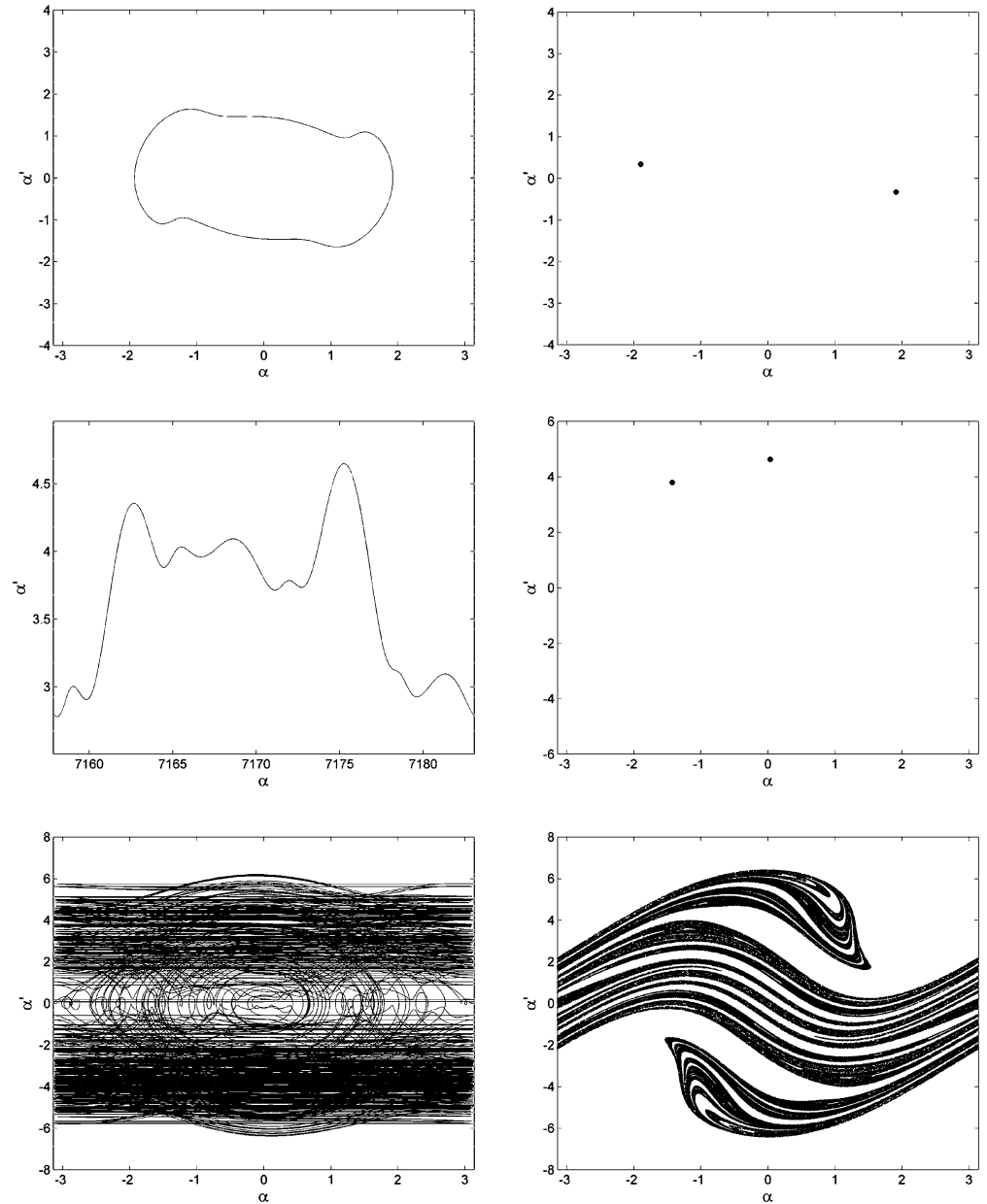

Fig. 14 Time history, phase portrait and Poincaré section for $\omega=1.8$ and $\varepsilon=0.9$. a $p=0.9, \mathbf{b} p=1.25, \mathbf{c} p=2.5, \mathbf{d} p=3.7$, e $p=4.5$

Fig. 11a. A period-1 pure rotation occurs in Fig. 11d and the chaotic attractor takes place in Fig. 11e.

For $\omega=1.8$ and $\varepsilon=0.4$ results are plotted in Fig. 12. As previously, the period-2 oscillation appears in Fig. 12a for $p$ equal to 0.95 . A chaotic attractor may be observed in Fig. $12 \mathrm{~b}$ and pure rotations can be seen in Fig. 12c, d, e. For $\omega=0.9$ and $\varepsilon=0.4$, period- 2 oscillations-rotations are in Fig. 13b, d. A chaotic attractor is present in Fig. 13c for $p$ equals 2.5 and a period-1 oscillation-rotation occurs in Fig. 13e. A pure oscillation period-1 is present in Fig. 13a.

Figure 14 brings the results for $\omega=1.8$ and $\varepsilon=0.9$, where chaotic attractors can be found in Fig. $14 \mathrm{c}$, e, but in different shapes. Period-2 pure rotations are in Fig. 14b, d. The period-2 oscillation attractor in Fig. 14a exhibits a more deformed orbit in comparison with the phase portrait in Fig. 10a.

Finally, for $\omega=0.9$ and $\varepsilon=0.9$, the obtained results are in Fig. 15. There is a period-1 oscillation in Fig. 15a and another in Fig. 15b. For $p$ equals 3.65 and 4.2 the dynamic behavior obtained was the period-2 oscillation-rotation. The chaotic attractor may be encountered for $p$ equals 3.0 in the Fig. 15c.

In a comparison among graphics from Figs. 10, 11, $12,13,14$ and 15, we can observe that pure rotational orbits were not found in Figs. 13 and 15, both with frequency in $\omega$ equals 0.9 , and $\varepsilon$ equals 0.4 and 0.9 , respectively. That is coherent with the results found in the "parameter plots", where one can observe that 
(d)

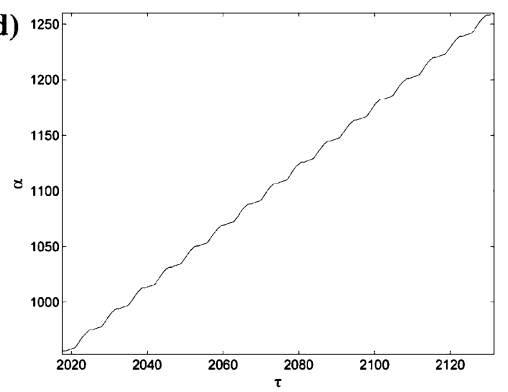

(e)

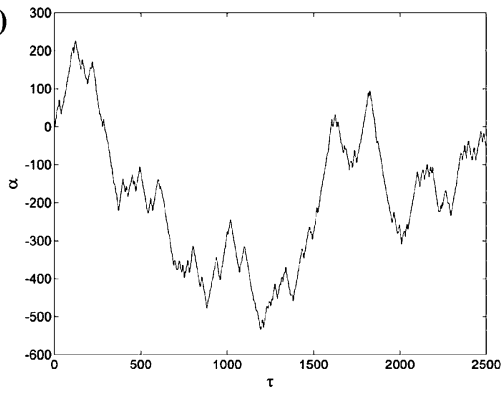

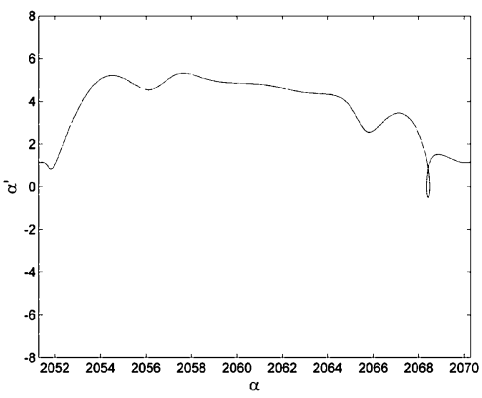
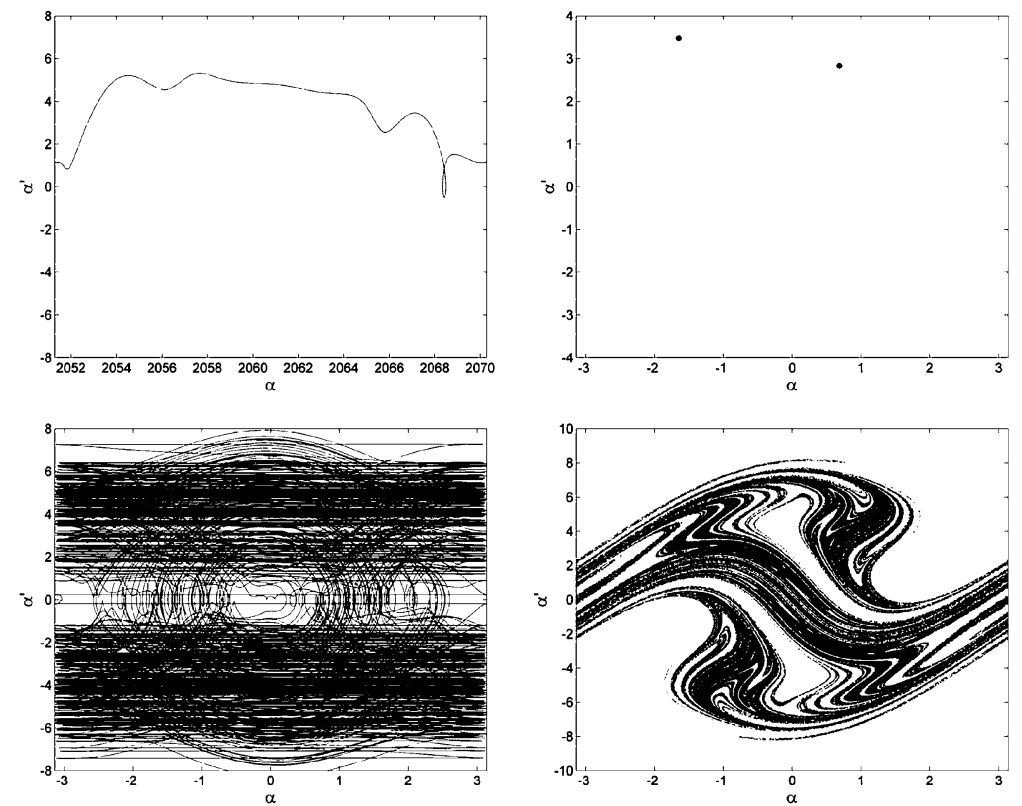

Fig. 14 continued

Table 2 Types of motion for values of the parameters $\omega$ and $\varepsilon$

\begin{tabular}{lll}
\hline & $\omega=1.8$ & $\omega=0.9$ \\
\hline$\varepsilon=0$ & Oscillation period-2 & Oscillation period-1 \\
& Rotation period-1 & Rotation period-1 \\
& Rotation period-2 & Oscillation-rotation period-2 \\
& Chaotic motion & Chaotic motion \\
& Oscillation period-2 & Oscillation period-1 \\
& Rotation period-1 & Oscillation-rotation period-1 \\
& Rotation period-2 & Oscillation-rotation period-2 \\
& Rotation period-4 & Chaotic motion \\
& Chaotic motion & \\
& Oscillation period-2 & Oscillation period-1 \\
& Oscillation-rotation period-2 & Oscillation-rotation period-2 \\
& Chaotic motion & Chaotic motion \\
\hline
\end{tabular}

rotational regions become narrower when the parameter $\varepsilon$ increases. In common, from Fig. 10 to the Fig. 15, chaotic motion occurs for all values of $\omega$ and $\varepsilon$ used when varying the parameter $p$. Only a change in the shape of the chaotic attractor was observed. In Table 2 the types of motion were enrolled relating with the parameters $\omega$ and $\varepsilon$ considering results encountered during the variation of $p$. 
(a)

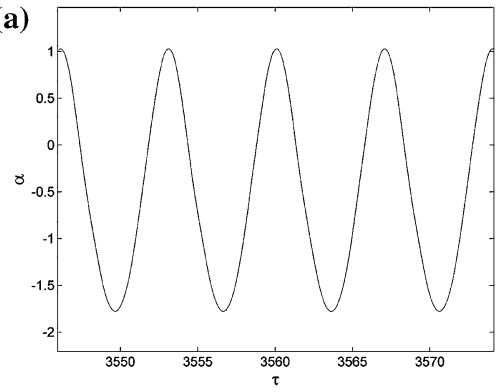

(b) 0

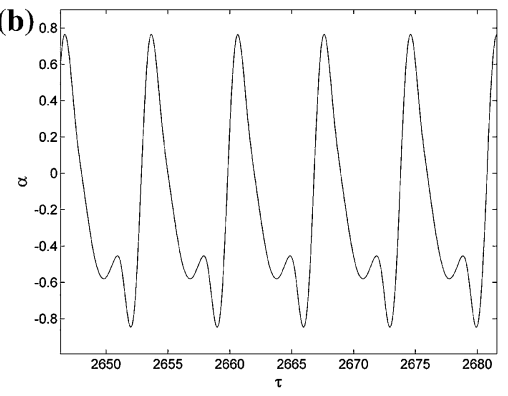

(c)

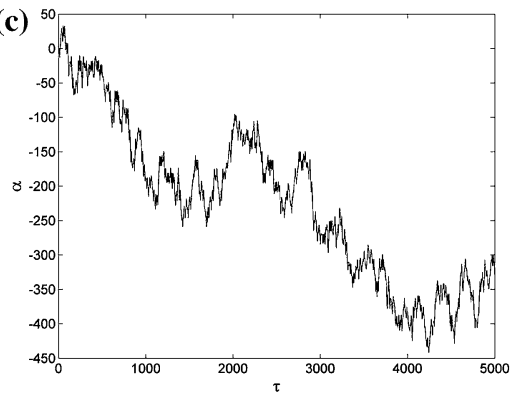

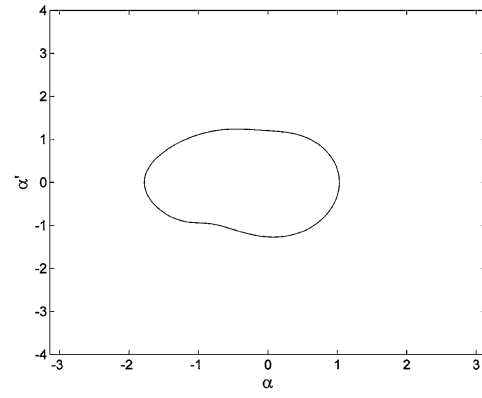
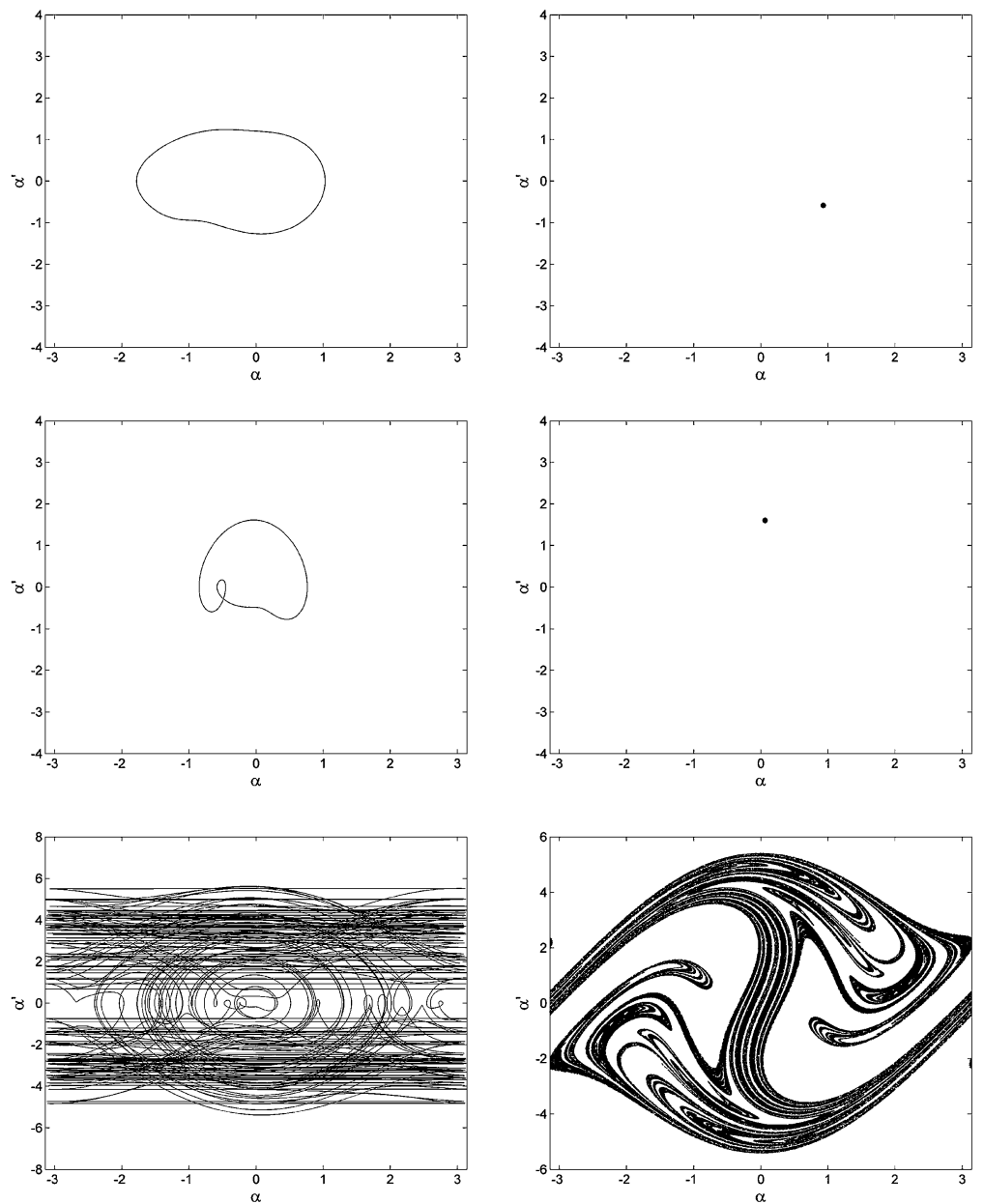

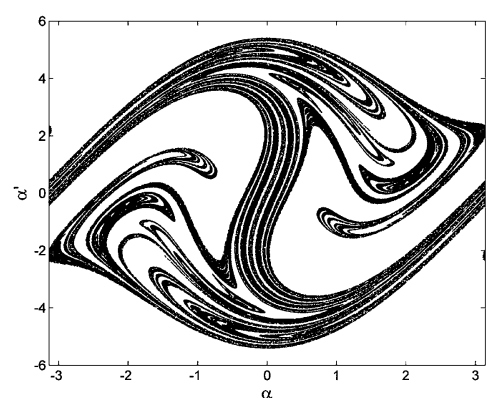

Fig. 15 Time history, phase portrait and Poincaré section for $\omega=0.9$ and $\varepsilon=0.9$. a $p=0.5$, b $p=1.9$, c $p=3.0$, d $p=3.65$, e $p=4.2$

\section{Conclusion}

The different kinds of motion take place for different values of $\varepsilon$. A greater value of $\varepsilon$ for $\omega=1.8$ provokes a clear increase of chaotic region in the bifurcation diagram. For the frequency $\omega=0.9$, an alteration of the $\varepsilon$ causes just a displacement of the chaotic interval.

However, the main difference is consisted in the increase of complexity observed on parameter plots and bifurcations diagrams. The parameter space plots for $\varepsilon$ equals 0.4 and 0.9 show an increase of uncertain regions in comparison with $\varepsilon$ equals zero. The bifurcation diagrams demonstrate the kinds of motion are associated with more narrow regions when $\varepsilon$ is bigger. In general, results become more complex as the value of $\varepsilon$ enlarges and the $\omega$ goes down.
The basin of attraction suggests the symmetry for the pendulum is maintained while varying the parameter $\varepsilon$. The opposite of what happens in the case of the nearly parametric pendulum of Horton et al. [30], when the value of the eccentricity of the elliptical excitation may cause a break of symmetry in the basin of attraction. Rotational regions in basin of attraction are present for different values of $\varepsilon$, however, the rotational regions shrink whilst the value of $\varepsilon$ gets bigger.

The future works aimed at addressing the main issues of the newly developed topic of dynamical integrity. The basic idea is that, sometimes, classical (Lyapunov) stability is not enough for practical purposes since there are cases in which the basin of attraction around a stable solution is so small to be 
(d)

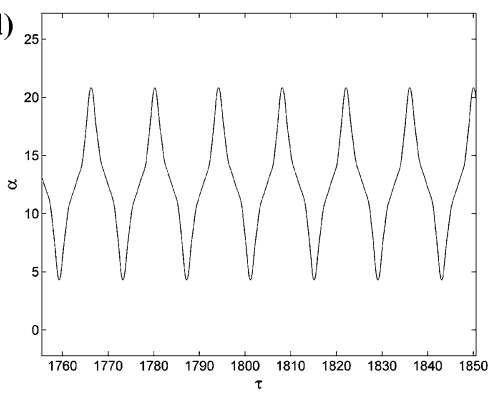

(e)

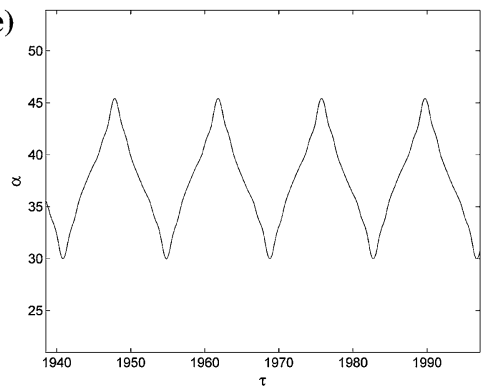

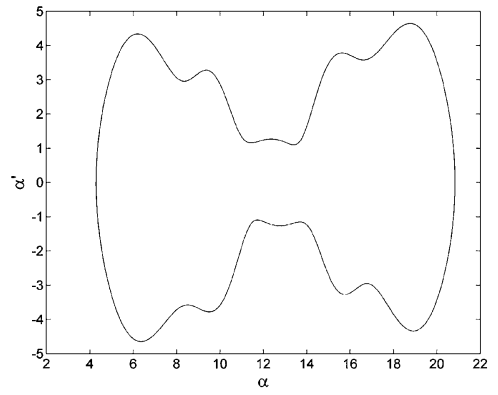
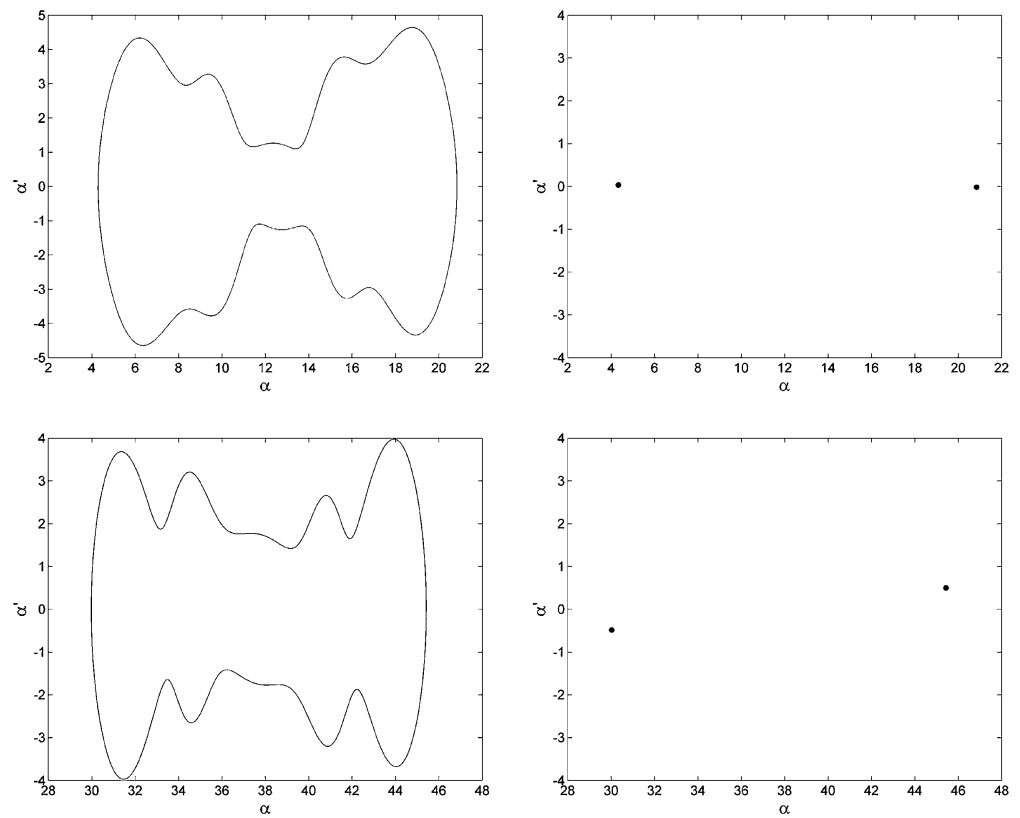

Fig. 15 continued

useless for practical purposes. This is particularly true close to bifurcations, and calls for a detailed analysis of basins structure and basins evolutions, which is the scope of dynamical integrity measures and erosion profiles. As demonstrated through the numerical results for certain parameters the system has a chaotic behavior, in order to keep the system on a periodic orbits previously defined, in future work will be considered the application of SDRE and OLFC controls. The SDRE (State Dependent Riccati Equation) and OLFC (Optimal Linear Feedback Control) techniques are recommended for nonlinear systems and successfully used in the suppression of chaotic behavior in the works [31-38] justifying its future application on the model studied in this work.

Acknowledgments The authors would like to acknowledge the Brazilian agencies: CNPq, FAPESP and CAPES, for the financial support.

\section{References}

1. Leven RW, Koch BP (1981) Chaotic behavior of a parametrically excited damped pendulum. Phys Lett A 86(2):71-74. doi:10.1016/0375-9601(81)90167-5

2. Clifford MJ, Bishop SR (1995) Rotating periodic orbits of the parametrically excited pendulum. Phys Lett A 201(2):191-196. doi:10.1016/0375-9601(95)00255-2
3. Clifford MJ, Bishop SR (1996) Locating oscillatory orbits of the parametrically-excited pendulum. J Aust Math Soc Ser B Appl Math 37(3):309-319. doi:10.1017/S03342700000 10687

4. Xu Xu, Wiercigroch M, Cartmell MP (2005) Rotating orbits of a parametrically-excited pendulum. Chaos Solitons Fractals 23(5):1537-1548. doi:10.1016/j.chaos.2004.06.053

5. Wiercigroch M (2005) A new concept of energy extraction from waves via parametric pendulor. UK patent application

6. Brasil RMLRF, Amaral L, Balthazar JM (2013) A crankshaft-slider energy harvester of sea waves. In: Proceedings of 14th Pan-American congress of applied mechanics, vol 1. Santiago, Chile, pp 35-36

7. Kecik K, Warminski J (2012) Chaos in mechanical pendulum-like system near main parametric resonance. In: Procedia IUTAM: IUTAM symposium on 50 years of chaos: applied and theoretical vol 5. pp 249-258. doi:10.1016/j. piutam.2012.06.034

8. Lenci S, Rega G (2011) Experimental versus theoretical robustness of rotating solutions in a parametrically excited pendulum: a dynamical integrity perspective. Phys D 240:814-824. doi:10.1016/j.physd.2010.12.014

9. Warminski J, Kecik K (2006) Autoparametric vibrations of a nonlinear system with pendulum. Math Prob Eng. doi:10. 1155/MPE/2006/80705

10. Warminski J, Kecik K (2009) Instabilities in the main parametric resonance area of a mechanical system with a pendulum. J Sound Vib 322(3):612-628. doi:10.1016/j.jsv. 2008.06.042

11. Lenci S, Pavlovskaia E, Rega G, Wiercigroch M (2008) Rotating solutions and stability of parametric pendulum by perturbation method. J Sound Vib 310:243-259. doi:10. 1016/j.jsv.2007.07.069 
12. Wang R, Jing Z (2004) Chaos control of chaotic pendulum system. Chaos Solitons Fractals 21:201-207. doi:10.1016/j. chaos.2003.10.011

13. Yokoi Y, Hikihara T (2011) Tolerance of start-up control of rotation in parametric pendulum by delayed feedback. Phys Lett A 375:1779-1783. doi:10.1016/j.physleta.2011.02.022

14. Lenci S, Brocchini M, Lorenzoni C (2012) Experimental rotations of a pendulum on water waves. J Comput Nonlinear Dyn 7(1):011007. doi:10.1115/1.4004547

15. Litak G, Wiercigroch M, Horton BW, Xu X (2010) Transient chaotic behavior versus periodic motion of a parametric pendulum by recurrence plots. Zamm J Appl Math Mech 90(1):33-41. doi:10.1002/zamm.200900290

16. Alevras P, Yurchenko D (2014) Naess A (2014) Stochastic synchronization of rotation of rotating parametric pendulums. Meccanica 49(8):1945-1954. doi:10.1007/s11012014-9955-4

17. Yang CC (2013) Synchronizations of rotating pendulums via self-learning terminal sliding-mode control subject to input nonlinearity. Nonlinear Dyn 72(3):695-705. doi:10. 1007/s11071-012-0746-y

18. de Paula AS, Savi MA, Wiercigroch M, Pavlovskaia E (2012) Bifurcation control of a parametric pendulum. Int J Bifurc Chaos. doi:10.1142/S0218127412501118

19. Szemplinska-Stupnicka W, Tyrkiel E (2002) Common features of the onset of the persistent chaos in nonlinear oscillators: a phenomenological approach. Nonlinear Dyn 27(3):271-293. doi:10.1023/A:1014456416158

20. Warminski J, Balthazar JM, Brasil RMLRF (2001) Vibrations of a non-ideal parametrically and self-excited model. J Sound Vib 245:363-374. doi:10.1006/jsvi.2000.3515

21. Lenci S, Rega G (2006) Dynamical integrity of nonlinear mechanical oscillators. In: Proceedings of the 16th European conference of fracture, book title fracture of nano and engineering materials and structures, book part: C. Alexandroupolis, Greece, pp 811-812. doi:10.1007/1-40204972-2_401

22. Belato D, Weber HI, Balthazar JM, Mook DT (2001) Chaotic vibrations of a nonideal eletro-mechanical system, 2001. Int J Solids Struct 38(10-13):1699-1706. doi:10. 1016/S0020-7683(00)00130-X

23. Belato D, Weber HI, Balthazar JM (2005) Using transient and steady state considerations to investigate the mechanism of loss of instability of a dynamical system. Appl Math Comput 164(2):605-613. In: 12th international workshop on dynamics and control. doi:10.1016/j.amc.2004.06.100

24. Hsieh SR, Shaw SW (1993) The Dynamic stability and nonlinear resonance of a flexible connecting rod: continuos parameter model. J Nonlinear Dyn 4(6):573-603. doi:10. 1007/BF00162233

25. Halbig D, Beale DG (1995) Experimental observations of a flexible slider crank mechanism at very high speeds. J Nonlinear Dyn 7(3):365-384. doi:10.1007/BF00046309

26. Wauer J, Bührle P (1997) Dynamics of a flexible slidercrank mechanism driven by a non-ideal source of energy.
J Nonlinear Dyn 13(3):221-242. doi:10.1023/A:10082103 10226

27. Goudas I, Stavrakis I, Natsiavas S (2004) Dynamics of slider-crank mechanisms with flexible supports and non-ideal forcing. J Nonlinear Dyn 35(3):205-227. doi:10.1023/B: NODY.0000027914.66360.01

28. Wolf A, Swift JB, Swinney HL, Vastano JA (1985) Determining Lyapunov exponents from a time series. J Phys D Nonlinear Phenom 16(3):285-317. doi:10.1016/01672789(85)90011-9

29. Butikov EI (1999) Parametric resonance. Comput Sci Eng 1(3):76-83. doi:10.1109/5992.764219

30. Horton B, Sieber J, Thompson JMT, Wiercigroch M (2011) Dynamics of a nearly parametric pendulum. Int J Nonlinear Mech 46(2):436-442. doi:10.1016/j.ijnonlinmec.2010.11. 003

31. Balthazar JM, Tusset AM, Souza SLTD, Bueno AM (2012) Microcantilever chaotic motion suppression in tapping mode atomic force microscope. In: Proceedings of the Institution of Mechanical Engineers. Part C. J Mech Eng Sci 227:1730-1741. DOI:10.1177/0954406212467933

32. Balthazar JM, Bassinello DG, Tusset AM, Bueno AM, Pontes BR Jr (2014) Nonlinear control in an electromechanical transducer with chaotic behavior. Meccanica 49(8):1859-1867. doi:10.1007/s11012-014-9910-4

33. Nozaki R, Balthazar JM, Tusset AM, Pontes BR Jr, Bueno AM (2013) Nonlinear control system applied to atomic force microscope including parametric errors. J Control Autom Electr Syst 24:223-231. doi:10.1007/s40313-0130034-1

34. Tusset AM, Piccirillo V, Bueno AM, Balthazar JM, Sado D, Felix JLP, Brasil RMLRD (2015) Chaos control and sensitivity analysis of a double pendulum arm excited by an RLC circuit based nonlinear shaker. J Vib Control 1:1-17. doi: $10.1177 / 1077546314564782$

35. Tusset AM, Balthazar JM, Chavarette FR, Felix JLP (2012) On energy transfer phenomena, in a nonlinear ideal and nonideal essential vibrating systems, coupled to a (MR) magneto-rheological damper. Nonlinear Dyn 69:1859-1880. doi:10.1007/s11071-012-0391-5

36. Tusset AM, Balthazar JM, Bassinello DG, Pontes BR Jr, Felix JLP (2012) Statements on chaos control designs, including a fractional order dynamical system, applied to a MEMS comb-drive actuator. Nonlinear Dyn 69:1837-1857. doi:10.1007/s11071-012-0390-6

37. Tusset AM, Bueno AM, Nascimento CB, Kaster MS, Balthazar JM (2013) Nonlinear state estimation and control for chaos suppression in MEMS resonator. Shock Vib 20:749-761. doi:10.3233/SAV-130782

38. Tusset AM, Balthazar JM, Felix JLP (2013) On elimination of chaotic behavior in a non-ideal portal frame structural system, using both passive and active controls. J Vib Control 19:803-813. doi:10.1177/1077546311435518 